\title{
Cultivar- and Wood Area-Dependent Metabolomic Fingerprints of Grapevine Infected by Botryosphaeria Dieback
}

\author{
Christelle Lemaitre-Guillier, ${ }^{1}$ Florence Fontaine, ${ }^{2}$ Chloé Roullier-Gall, ${ }^{3}$ Mourad Harir, ${ }^{4,5}$ Maryline Magnin-Robert, ${ }^{2}$ \\ Christophe Clément, ${ }^{2}$ Sophie Trouvelot, ${ }^{1}$ Régis D. Gougeon, ${ }^{3}$ Philippe Schmitt-Kopplin,,${ }^{4,5}$ and Marielle Adrian ${ }^{1, \dagger}$ \\ ${ }^{1}$ Agroécologie, AgroSup Dijon, CNRS, INRAE, Univ. Bourgogne, Univ. Bourgogne Franche-Comté, F-21000 Dijon, France \\ ${ }^{2}$ SFR Condorcet FR CNRS3417, Université de Reims Champagne-Ardenne, RIBP EA 4707, Laboratoire Résistance Induite et Bioprotection \\ des Plantes, BP 1039, 51687 Reims Cedex 2, France \\ ${ }^{3}$ UMR PAM Université de Bourgogne/AgroSup Dijon, Institut Universitaire de la Vigne et du Vin, Jules Guyot, Dijon, France \\ ${ }^{4}$ Technische Universität München, Chair of Analytical Food Chemistry, Freising-Weihenstephan, Germany \\ ${ }^{5}$ Research Unit Analytical BioGeoChemistry, Helmholtz Zentrum München, German Research Center for Environmental Health, Neuherberg, \\ Germany \\ Accepted for publication 21 June 2020.
}

ABSTRACT

\begin{abstract}
Botryosphaeria dieback is one of the most significant grapevine trunk diseases that affects the sustainability of the vineyards and provokes economic losses. The causal agents, Botryosphaeriaceae species, live in and colonize the wood of the perennial organs causing wood necrosis. Diseased vines show foliar symptoms, chlorosis, or apoplexy, associated to a characteristic brown stripe under the bark. According to the susceptibility of the cultivars, specific proteins such as PR-proteins and other defense-related proteins are accumulated in the brown stripe compared with the healthy woody tissues. In this study, we enhanced the characterization of the brown stripe and the healthy wood by obtaining a metabolite profiling for the three cultivars Chardonnay, Gewurztraminer,
\end{abstract}

and Mourvèdre to deeper understand the interaction between the Botryosphaeria dieback pathogens and grapevine. The study confirmed a specific pattern according to the cultivar and revealed significant differences between the brown stripe and the healthy wood, especially for phytochemical and lipid compounds. This is the first time that such chemical discrimination was made and that lipids were so remarkably highlighted in the interaction of Botryosphaeriaceae species and grapevine. Their role in the disease development is discussed.

Keywords: Botryosphaeria dieback, cultivar, FC-ICR-MS, grapevine trunk diseases, lipids, metabolomics, phytochemicals
Grapevine trunk diseases (GTDs), namely Esca, Botryosphaeria, and Eutypa diebacks are now considered as a major concern in viticulture. In France, a survey of 329 vineyards during the 2003 to 2008 period revealed that 54 to $100 \%$ of them were affected by Esca/Botryosphaeria dieback, depending on the region and year (Bruez et al. 2013). In 2008, about 2 to $8 \%$ of vines expressed symptoms (Bruez et al. 2013). In Spain, the incidence of GTDs was 10.5\% in 2007 (Rubio and Garzón 2011) and reached 60 to $80 \%$ in old south Italian vineyards (Romanazzi 2009). About $13 \%$ of the French vineyard was considered unproductive in 2012, because of GTDs, and it reached 20\% in Spain and 30\% in Italy (Grosman and Doublet 2012). GTDs still extend worldwide and gradually degrade the vineyards, leading to huge economic impact due to yield decrease and vine death (Guerin-Dubrana et al. 2019). Costs for replacement of dead vines were indeed estimated to at least 1.5 billion dollars per year (Hofstetter et al. 2012). In California, overall costs associated to GTDs were estimated at more than $\$ 260$ million per year (for review, see Fontaine et al. 2016).

GTDs are caused by xylem-inhabiting fungi that colonize and degrade wood tissues, with subsequent symptoms on the aerial parts. Twenty-one different fungi of Botryosphaeriaceae species were associated to Botryosphaeria dieback; among with Neofusicoccum

†Corresponding author: M. Adrian; marielle.adrian@u-bourgogne.fr

*The $e$-Xtra logo stands for "electronic extra" and indicates that four supplementary figures and supplementary materials are published online.

The author(s) declare no conflict of interest.

(c) 2020 The American Phytopathological Society parvum, Botryosphaeria dothidea, and Diplodia seriata (UrbezTorres 2011; van Niekerk et al. 2006). Symptoms of Botryosphaeria dieback on the aerial parts consist in leaf intervein coloration or leaf wilting, bud necrosis or mortality, dead arms, shoot dieback, and bunch rot (Bertsch et al. 2013; Larignon et al. 2001; Spagnolo et al. 2014; Urbez-Torres 2011; van Niekerk et al. 2004). At the trunk level, the fungi cause perennial cankers, brown wood streaking, internal necrotic lesions, or a typical longitudinal orange/brown stripe located in the outer xylem, superficially and just beneath the bark (Fontaine et al. 2016; Larignon et al. 2001; Urbez-Torres 2011). This longitudinal stripe has the particularity to be undetectable before the vegetative season and is therefore associated with leaf symptoms (Larignon et al. 2001).

Although cultivars of Vitis vinifera $\mathrm{L}$. could be ranked on the basis of the degree of GTD symptom expression (Bruez et al. 2013; Grosman and Doublet 2012; Murolo and Romanazzi 2014), none of them was found to be resistant. For example, Chardonnay is considered as less susceptible, Mourvèdre or Gewurztraminer susceptible, and Savagnin highly susceptible to Esca disease/ Botryosphaeria dieback (Grosman and Doublet 2012). Despite active research, no effective and generalizable strategies of crop protection are now available (Mondello et al. 2018a, b), which makes further investigation necessary. Getting a good knowledge of the effects of the disease on the plant can help identify ways to limit its impact. Few years ago, a proteomic study was done to compare the response of woody tissues to Botryosphaeria dieback of the three cultivars Chardonnay, Mourvèdre, and Gewurztraminer, localized on the longitudinal brown stripe (Spagnolo et al. 2014). Accumulation of several proteins (PR proteins, and other stress- and defense-related proteins like polyphenol oxidase) were found inside the brown stripe wood of diseased vines compared with the neighboring apparent healthy wood of diseased or control vines. 
Moreover, the differential of the susceptibility of the three cultivars could be linked to the diverse expression of proteins. In the present study, the same samples were analyzed by an ultra-high resolution method of metabolome analysis (Fourier transform-ion cyclotron resonance-mass spectrometry [FT-ICR-MS]), to obtain a metabolite profiling of these different woody areas, for the three cultivars, and to progress in the understanding of the impact of Botryosphaeria dieback in grapevine.

\section{MATERIALS AND METHODS}

Plant material. It was the same sampling as the one described in Spagnolo et al. (2014), i.e., Chardonnay, Gewurztraminer (white cultivars), and Mourvèdre (red cultivar). For each of them, three asymptomatic (control) and three Botryosphaeria dieback expressing vines, respectively, were uprooted in July 2011 from three French vineyards to collect samples. Control plants did not express any GTD-related foliar symptom since at least 5 years. Three groups of samples were collected per cultivar: asymptomatic white trunk wood from control (AC) and diseased (AD) plants, and brown striped trunk wood from diseased (SD) plants (Supplementary Fig. S1).

A total of nine samples corresponding to three biological replicates per group (i.e., three wood samples from three separated plants) were used for analysis. Raw wood samples collected in 2011 were immediately frozen in the field with liquid nitrogen and subsequently stored at $-80^{\circ} \mathrm{C}$ until metabolomics analyses. In 2013 , frozen wood samples were ground to a fine powder in liquid nitrogen with a Mixer Mill MM 400 (Retsch, Haan, Germany), and powders were then extracted for FT-ICR-MS metabolomics analyses.

FT-ICR-MS analysis. Prior to analysis, $15 \mathrm{mg}$ of each sample were extracted with $1 \mathrm{ml}$ of methanol (LC-MS grade, Fluka Analytical; Sigma-Aldrich, St. Louis, MO, U.S.A.) and sonicated for $30 \mathrm{~min}$. After centrifugation $(25,000 \times \mathrm{g}, 10 \mathrm{~min}$, room temperature), the supernatant was collected and rediluted in methanol (1/50 [vol/vol]). Ultra-high resolution mass spectra were acquired using an FT-ICR-MS (solariX, Bruker Daltonics GmbH, Bremen, Germany) equipped with a 12 Tesla superconducting magnet (Magnex Scientific Inc., Yarnton, GB) and an APOLO II ESI source (Bruker Daltonics $\mathrm{GmbH}$ ) operated in the negative ionization mode. Samples were introduced into the microelectrospray source at a flow rate of $120 \mu \mathrm{l} / \mathrm{h}$. Spectra were acquired with a time domain of 4 mega words over an $\mathrm{m} / \mathrm{z}$ mass range of 100 to 1,000 , and 300 scans were accumulated per sample.

Data analysis. Spectra were externally calibrated on clusters of arginine (methanol at $10 \mathrm{mg} /$ liter). Further internal calibration was

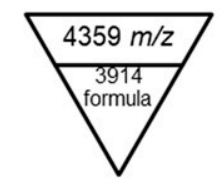

CH

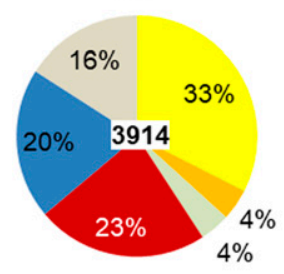

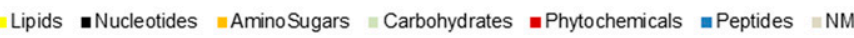

Fig. 1. Characteristics of the three cultivar subsets. The global data file was divided into three cultivar subsets. Values on the top of the figure correspond to the number of raw $\mathrm{m} / \mathrm{z}$ and assigned formulas for each cultivar. The structural categories were next defined by multidimensional stoichiometric constraints classification on the validated $\mathrm{m} / \mathrm{z}$, subsets and relative proportions illustrated on pie plots. $\mathrm{CH}$, Chardonnay; GW, Gewurztraminer; MV, Mourvèdre; and NM, nonmatching structures. performed for each sample by using a list of ubiquitous fatty acids and recurrent wine compounds, allowing mass accuracies of 0.1 ppm (Gougeon et al. 2009). After acquisition, all $\mathrm{m} / \mathrm{z}$ detected were given an identification number (ID) in order to simplify further data processing. Data treatments were applied on the $\mathrm{m} / \mathrm{z}$ lists and detailed in Supplementary Figure S2. First, $\mathrm{m} / z$ were reduced to values seen at least three times either in the whole data set or in the cultivar subsets. Remaining masses $(\mathrm{m} / \mathrm{z}$ ) coupled to their respective peak intensities were submitted to statistical analysis with the Perseus software (http://maxquant.net/perseus/), which performed hierarchical clustering (HCA) and multivariate analysis of variance (ANOVA) $(P<0.05)$ between groups of samples to get significant $m / z$ (Supplementary Fig. S2, step a). $m / z$ were also subjected to the NetCalc algorithm and in-house software tools to obtain elemental
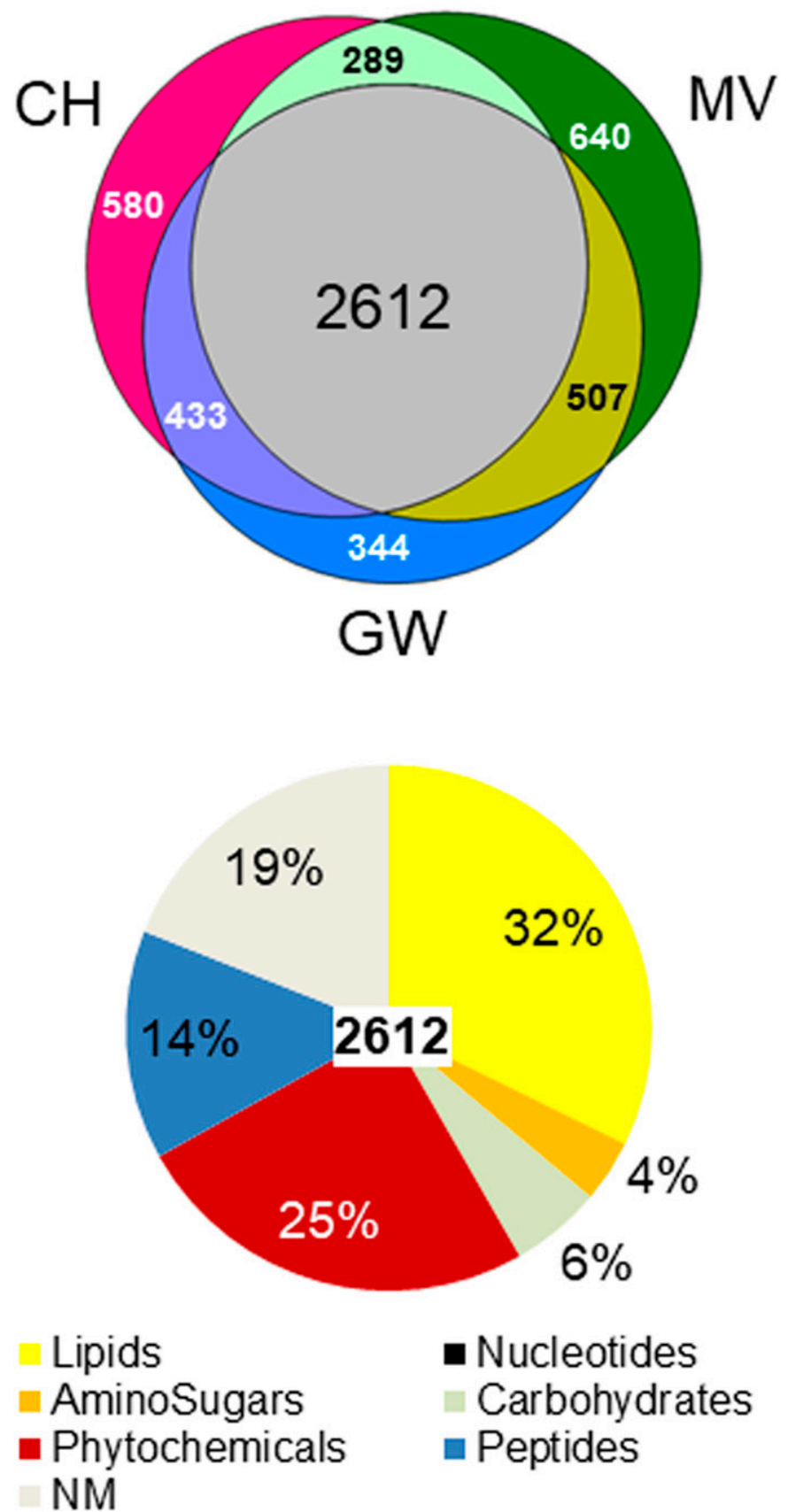

Fig. 2. Comparison of the three cultivar data subsets. Venn diagram of the distribution of specific formulas for the three cultivars (top panel) $(\mathrm{CH}$, Chardonnay; GW, Gewurztraminer; and MV, Mourvèdre) and multidimensional stoichiometric constraints classification structural categories distribution of the 2,612 common formulas (bottom panel). NM, nonmatching structures. 


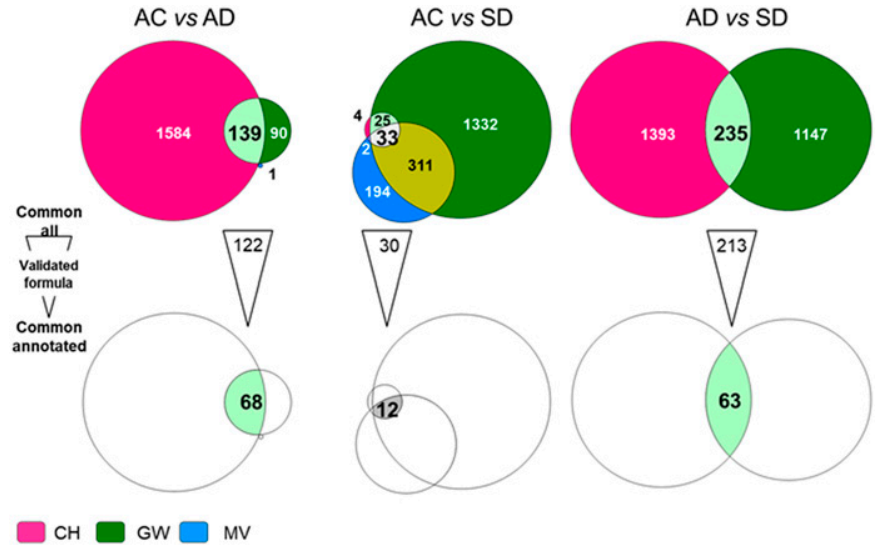

Fig. 3. Comparison of $\mathrm{AC}, \mathrm{AD}$, and $\mathrm{SD}$ sample types. For each comparison, Venn diagrams at the top of the illustration indicate the number of differentially accumulated $\mathrm{m} / \mathrm{z}$, common/specific to the three cultivars. The common $\mathrm{m} / \mathrm{z}$ were next validated (number indicated in the middle part of the figure) and submitted to search for identification (MassTrix). Resulting numbers of annotated $\mathrm{m} / \mathrm{z}$ are indicated in the bottom Venn diagrams. AC, white wood of asymptomatic vines; $\mathrm{AD}$, white wood of diseased vines; $\mathrm{SD}$, brown stripe of diseased vines; $\mathrm{CH}$, Chardonnay; GW, Gewurztraminer; MV, Mourvèdre; and $\mathrm{NM}$, nonmatching structures. chemical formula (Tziotis et al. 2011) validated by setting van Krevelen $(\mathrm{VK})$ chemical constraints $(\mathrm{O} / \mathrm{C}$ ratio $\leq 1$; double bound equivalent (DBE): $0<\mathrm{DBE} / \mathrm{C}<5 \mathrm{H} / \mathrm{C}$ ratio $\leq 2 \mathrm{n}+2$ and aromaticity index (IA): $-20<$ IA $<0.7$; element counts: $\mathrm{C} \leq 100, \mathrm{H} \leq 200, \mathrm{O} \leq$ $80, n \leq 3, \mathrm{~S} \leq 3$, and $P \leq 1$ ) applied to exclude rare or impossible formulas (Supplementary Fig. S2, step b). Elemental formulas were next classified into the main putative chemical functional categories (lipids, peptides, amino sugars, carbohydrates, nucleotides, and phytochemicals/aromatic compounds) using the multidimensional stoichiometric constraints classification (MSCC) described by Rivas-Ubach et al. (2018), based on the C/H/O/N/P stoichiometric ratios (Supplementary Fig. S2, step c). We used the short term "phytochemicals" for "phytochemicals/aromatic compounds," which corresponds to secondary metabolites. Proportions of resulting predicted classified and nonclassified (not matching: NM) categories are graphically represented on pie plots. $\mathrm{m} / \mathrm{z}$ values were also fed into MassTrix software (http://masstrix3.helmholtzmuenchen.de/masstrix3/) for annotation requests using KEGG, HMDB, and LipidMaps databases, and $V$. vinifera as preferred organism (1 ppm tolerance value) (Supplementary Fig. S2, step d). Compounds identified as isotopologues $\left({ }^{13} \mathrm{C},{ }^{15} \mathrm{~N}\right.$, and $\left.{ }^{18} \mathrm{O}\right)$ already listed helped for category revision when necessary (MSCC, Supplementary Fig. S2, step e) but were discarded from output lists and further discussions. In pairwise comparisons, the most

TABLE 1. List of the 23 top $m / z$ accumulated in Chardonnay AD samples compared with AC samples ${ }^{\mathrm{a}}$

\begin{tabular}{|c|c|c|c|c|c|c|c|}
\hline ID & $P$ & $t$ test & Formula & Cat. & $m / z$ & \multicolumn{2}{|c|}{ Annotations } \\
\hline 6398 & 0.0001 & -6.119 & $\mathrm{C}_{7} \mathrm{H}_{13} \mathrm{NO}_{5}$ & As & 190.072108 & 2-Amino-3,7-dideoxy-D-threo-hept-6-ulosoni & acid \\
\hline 10556 & 0.0015 & -4.668 & $\mathrm{C}_{18} \mathrm{H}_{14} \mathrm{O}_{8}$ & $\mathrm{Ph}$ & 357.061607 & $\begin{array}{l}5,3^{\prime} \text {-Dihydroxy- } 4^{\prime}, 5^{\prime} \text {-dimethoxy- } 6,7- \\
\text { methylenedioxyisoflavone }\end{array}$ & Dalpalatin \\
\hline 7059 & 0.0004 & -4.473 & $\mathrm{C}_{9} \mathrm{H}_{14} \mathrm{~N}_{2} \mathrm{O}_{5}$ & $\mathrm{Pp}$ & 229.082987 & $\begin{array}{l}\text { Aspartyl-L-proline; }(2 S)-1-[(2 S)-2 \text {-amino-3-c } \\
\text { acid }\end{array}$ & rboxy-propanoyl]pyrrolidine-2-carboxylic \\
\hline 10808 & 0.0001 & -4.363 & $\mathrm{C}_{24} \mathrm{H}_{46} \mathrm{O}_{2}$ & Lp & 365.342494 & (15Z)-Tetracosenoic acid; nervonic acid & 10,13-Dimethyl-11-docosyne-10,13-diol \\
\hline 6580 & $<0.0001$ & -4.254 & $\mathrm{C}_{11} \mathrm{H}_{12} \mathrm{~N}_{2} \mathrm{O}_{2}$ & $\mathrm{Pp}$ & 203.082615 & $\begin{array}{l}\text { L-Tryptophan; tryptophan; }(\mathrm{S}) \text { - } \alpha \text {-amino- } \\
\beta \text {-(3-indolyl)-propionic acid }\end{array}$ & D-Tryptophan \\
\hline 6805 & 0.0025 & -4.207 & $\mathrm{C}_{9} \mathrm{H}_{17} \mathrm{NO}_{5}$ & Lp & 218.103393 & $\begin{array}{l}\text { Pantothenate; pantothenic acid; } \\
\text { (R)-pantothenate }\end{array}$ & $\begin{array}{l}\text { Pantothenic acid (see KEGG C00864); } \\
\text { 3-[[(2R)-2,4-dihydroxy-3,3-dimethyl- } \\
\text { butanoyl]amino]propanoic acid }\end{array}$ \\
\hline 8781 & 0.0005 & -4.134 & $\mathrm{C}_{19} \mathrm{H}_{38} \mathrm{O}_{2}$ & Lp & 297.279901 & Nonadecanoic acid; nonadecylic acid & Tuberculostearic acid \\
\hline 9077 & 0.0008 & -4.130 & $\mathrm{C}_{18} \mathrm{H}_{28} \mathrm{O}_{4}$ & Lp & 307.191489 & 5-O-methylembelin & \\
\hline 8030 & 0.0003 & -3.952 & $\mathrm{C}_{17} \mathrm{H}_{34} \mathrm{O}_{2}$ & Lp & 269.248606 & Methyl palmitate & Heptadecanoic acid \\
\hline 8390 & $<0.0001$ & -3.942 & $\mathrm{C}_{12} \mathrm{H}_{15} \mathrm{NO}_{7}$ & $\mathrm{Ph}$ & 284.077572 & Buchananine & \\
\hline 11523 & 0.0001 & -3.938 & $\mathrm{C}_{22} \mathrm{H}_{16} \mathrm{O}_{7}$ & $\mathrm{Ph}$ & 391.082331 & Hosloppin & 8-C-p-hydroxybenzylkaempferol \\
\hline 6352 & 0.0030 & -3.901 & $\mathrm{C}_{8} \mathrm{H}_{13} \mathrm{NO}_{4}$ & Lp & 186.077178 & 6-Acetamido-3-oxohexanoate & $\begin{array}{l}\text { 6-Acetamido-2-oxohexanoate; } 2 \text {-Oxo-6- } \\
\text { acetamidocaproate }\end{array}$ \\
\hline 6569 & 0.0017 & -3.895 & $\mathrm{C}_{9} \mathrm{H}_{17} \mathrm{NO}_{4}$ & Lp & 202.108462 & L-Acetylcarnitine (see KEGG C02571); (3S) & 3-acetyloxy-4-trimethylammonio-butanoate \\
\hline 9192 & 0.0006 & -3.89 & $\mathrm{C}_{20} \mathrm{H}_{40} \mathrm{O}_{2}$ & Lp & 311.295532 & Phytanate; phytanic acid & \\
\hline 7693 & 0.0024 & -3.803 & $\mathrm{C}_{11} \mathrm{H}_{20} \mathrm{~N}_{2} \mathrm{O}_{5}$ & $\mathrm{Pp}$ & 259.129924 & L- $\gamma$-Glutamyl-L-isoleucine & L- $\gamma$-Glutamyl-L-leucine \\
\hline 10447 & 0.0009 & -3.763 & $\mathrm{C}_{20} \mathrm{H}_{32} \mathrm{O}_{5}$ & $\mathrm{Lp}$ & 351.2177 & $\begin{array}{l}\text { Prostaglandin } \mathrm{H} 2 ;(5 \mathrm{Z}, 13 \mathrm{E})-(15 \mathrm{~S})-9 \alpha, 11 \alpha- \\
\text { epidioxy-15-hydroxyprosta-5,13-dienoate; } \\
\text { (5Z,13E,15S)-9 } \alpha, 11 \alpha \text {-epidioxy-15- } \\
\text { hydroxyprosta-5,13-dienoate }\end{array}$ & $\begin{array}{l}\text { Prostaglandin E2; (5Z,13E)-(15S)-11 } \alpha, 15 \text { - } \\
\text { dihydroxy-9-oxoprosta-5,13-dienoate; } \\
\text { (5Z,13E)-(15S)-11 } \alpha, 15 \text {-dihydroxy-9- } \\
\text { oxoprost-13-enoate; dinoprostone }\end{array}$ \\
\hline 6364 & 0.0038 & -3.732 & $\mathrm{C}_{9} \mathrm{H}_{16} \mathrm{O}_{4}$ & Lp & 187.097585 & $\begin{array}{l}\text { Azelaic acid; nonanedioic acid; 1,7- } \\
\text { heptanedicarboxylic acid }\end{array}$ & $\begin{array}{l}\text { Azelaic acid (see KEGG C08261); } \\
\text { nonanedioic acid }\end{array}$ \\
\hline 10007 & 0.0015 & -3.731 & $\mathrm{C}_{22} \mathrm{H}_{44} \mathrm{O}_{2}$ & Lp & 339.326849 & Docosanoic acid; docosanoate; behenic acid & $\begin{array}{l}\text { Behenic acid (see KEGG C08281); } \\
\text { docosanoic acid }\end{array}$ \\
\hline 8715 & 0.0053 & -3.72 & $\mathrm{C}_{18} \mathrm{H}_{32} \mathrm{O}_{3}$ & $\mathrm{Lp}$ & 295.227864 & $\begin{array}{l}\text { (9Z,12Z)-(8S)-Hydroxyoctadeca-9,12- } \\
\text { dienoic acid; laetisaric acid; } \\
\text { 8-hydroxylinoleic acid }\end{array}$ & $\begin{array}{l}\text { (9Z)-(12S,13R)-12,13-Epoxyoctadecenoic } \\
\text { acid; vernolic acid; cis-12,13-epoxy-cis-9- } \\
\text { octadecenoic acid }\end{array}$ \\
\hline 7362 & 0.0027 & -3.69 & $\mathrm{C}_{13} \mathrm{H}_{24} \mathrm{O}_{4}$ & Lp & 243.160178 & $\begin{array}{l}\text { 1,11-Undecanedicarboxylic acid; } \\
\text { tridecanedioic acid }\end{array}$ & 2-Methyl-dodecanedioic acid \\
\hline 6674 & 0.0009 & -3.661 & $\mathrm{C}_{11} \mathrm{H}_{14} \mathrm{O}_{4}$ & $\mathrm{Ph}$ & 209.08194 & Sinapyl alcohol; sinapoyl alcohol & $\begin{array}{l}\text { 3-Methyl-1-(2,4,6-trihydroxyphenyl)butan- } \\
\text { 1-one }\end{array}$ \\
\hline 7602 & 0.0008 & -3.639 & $\mathrm{C}_{16} \mathrm{H}_{32} \mathrm{O}_{2}$ & $\mathrm{Lp}$ & 255.232949 & $\begin{array}{l}\text { Hexadecanoic acid; hexadecanoate; } \\
\text { hexadecylic acid; palmitic acid; palmitate; } \\
\text { cetylic acid }\end{array}$ & $\begin{array}{l}\text { Palmitic acid (see KEGG C00249); } \\
\text { hexadecanoic acid }\end{array}$ \\
\hline 8073 & 0.0035 & -3.624 & $\mathrm{C}_{16} \mathrm{H}_{32} \mathrm{O}_{3}$ & Lp & 271.227874 & $\begin{array}{l}\text { (R)-3-Hydroxy-hexadecanoic acid; (3R)-3- } \\
\text { hydroxyhexadecanoic acid }\end{array}$ & $\begin{array}{l}\text { 2-Hydroxy palmitic acid; 2-hydroxy- } \\
\text { hexadecanoic acid }\end{array}$ \\
\hline
\end{tabular}

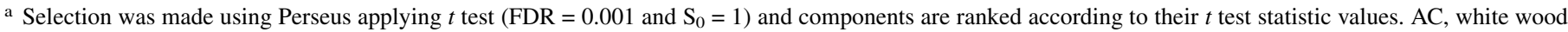
of asymptomatic vines; AD, white wood of diseased vines. ID, component identity; $P$, $t$ test associated $P$ value; Cat., multidimensional stoichiometric constraints classification (MSCC) predicted structural category; amino sugars (As); phytochemicals (Ph); peptides (Pp); lipids (Lp); carbohydrates (Cb); and $m / z$, measured mass value. 


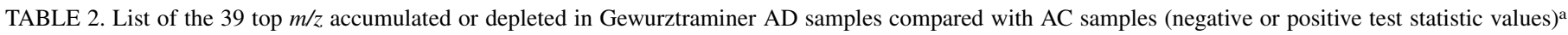

\begin{tabular}{|c|c|c|c|c|c|c|c|}
\hline ID & $P$ & $t$ test & Formula & Cat. & $m / z$ & Anno & ations \\
\hline 6371 & 0.0328 & -2.951 & $\mathrm{C}_{7} \mathrm{H}_{11} \mathrm{NO}_{5}$ & As & 188.056451 & $\begin{array}{l}\text { N-Acetyl-L-glutamate; N-acetyl-L-glutamic } \\
\text { acid }\end{array}$ & $\begin{array}{l}\text { L-2-Amino-6-oxoheptanedioate; L-2-amino- } \\
\text { 6-oxopimelate }\end{array}$ \\
\hline 9942 & 0.0106 & -2.909 & $\mathrm{C}_{16} \mathrm{H}_{18} \mathrm{O}_{8}$ & $\mathrm{Ph}$ & 337.092839 & 1-Caffeoyl-4-deoxyquinic acid & 4-p-Coumaroylquinic acid \\
\hline 12506 & 0.0112 & -2.901 & $\mathrm{C}_{21} \mathrm{H}_{37} \mathrm{O}_{7} \mathrm{P}$ & Lp & 431.220428 & $\begin{array}{l}\text { PA(18:3(6Z,9Z,12Z)/0:0); 1-(6Z,9Z,12Z- } \\
\text { octadecatrienoyl)-glycero-3P }\end{array}$ & $\begin{array}{l}\mathrm{PA}(18: 3(9 \mathrm{Z}, 12 \mathrm{Z}, 15 \mathrm{Z}) / 0: 0) ; 1-(9 \mathrm{Z}, 12 \mathrm{Z}, 15 \mathrm{Z}- \\
\text { octadecatrienoyl)-glycero-3P }\end{array}$ \\
\hline 8729 & 0.0291 & -2.901 & $\mathrm{C}_{14} \mathrm{H}_{19} \mathrm{NO}_{6}$ & Lp & 296.113986 & $\begin{array}{c}\text { Phenethylamine glucuronide (see KEGG C03 } \\
\text { phenylethylamino)oxane-2-carboxylic acid }\end{array}$ & $33) ;(2 \mathrm{~S}, 3 \mathrm{~S}, 4 \mathrm{~S}, 5 \mathrm{R}, 6 \mathrm{R})-3,4,5$-trihydroxy-6-(2- \\
\hline 13981 & 0.0157 & -2.855 & $\mathrm{C}_{21} \mathrm{H}_{24} \mathrm{O}_{13}$ & $\mathrm{Ph}$ & 483.114396 & Diospyrin & \\
\hline 8447 & 0.0089 & -2.852 & $\mathrm{C}_{12} \mathrm{H}_{17} \mathrm{NO}_{7}$ & $\mathrm{Lp}$ & 286.093239 & Volkenin & \\
\hline 6414 & 0.0087 & -2.768 & $\mathrm{C}_{7} \mathrm{H}_{12} \mathrm{O}_{6}$ & $\mathrm{Cb}$ & 191.056114 & $\begin{array}{l}\text { Quinate; quinic acid; kinic acid; chinic acid; } \\
\text { L-quinic acid; L-quinate; (-)-quinic acid }\end{array}$ & $\begin{array}{l}\text { 2D-5-O-methyl-2,3,5/4,6- } \\
\text { pentahydroxycyclohexanone; 5D-5-O- } \\
\text { methyl-2,3,5/4,6- } \\
\text { pentahydroxycyclohexanone; D-5-O- } \\
\text { methyl-2,3,5/4,6- } \\
\text { pentahydroxycyclohexanone }\end{array}$ \\
\hline 13994 & 0.0102 & -2.743 & $\mathrm{C}_{22} \mathrm{H}_{45} \mathrm{O}_{9} \mathrm{P}$ & Lp & 483.272897 & PG(16:0/0:0); 1-hexadecanoyl-sn-glycero-3-p & ospho-(1'-sn-glycerol) \\
\hline 17801 & 0.0277 & -2.732 & $\mathrm{C}_{42} \mathrm{H}_{79} \mathrm{O}_{13} \mathrm{P}$ & Lp & 821.518937 & $\begin{array}{l}\text { PI(13:0/20:1(11Z)); 1-tridecanoyl-2- } \\
\quad(11 Z \text {-eicosenoyl)-glycero-3-phospho- } \\
\left(1^{\prime} \text {-myo-inositol) }\right.\end{array}$ & $\begin{array}{l}\text { PI(14:0/19:1(9Z)); 1-tetradecanoyl-2- } \\
\text { (9Z-nonadecenoyl)-glycero-3-phospho- } \\
\text { (1'-myo-inositol) }\end{array}$ \\
\hline 17738 & 0.0158 & -2.727 & $\mathrm{C}_{43} \mathrm{H}_{75} \mathrm{O}_{12} \mathrm{P}$ & Lp & 813.492787 & $\begin{array}{l}\text { PI(P-16:0/18:4(6Z,9Z,12Z,15Z)); 1-(1Z-hexac } \\
\text { octadecatetraenoyl)-glycero-3-phospho-(1'-1 } \\
\text { acylglycerophosphoinositols] }\end{array}$ & $\begin{array}{l}\text { ecenyl)-2-(6Z,9Z,12Z,15Z- } \\
\text { yo-inositol) [1Z-alkenyl,2- }\end{array}$ \\
\hline 15389 & 0.0280 & -2.715 & $\mathrm{C}_{25} \mathrm{H}_{34} \mathrm{O}_{14}$ & Lp & 557.187735 & 7-Epiloganin tetraacetate & \\
\hline 16418 & 0.0169 & -2.714 & $\mathrm{C}_{36} \mathrm{H}_{65} \mathrm{O}_{8} \mathrm{P}$ & Lp & 655.434639 & $\begin{array}{l}\text { PA(13:0/20:3(8Z,11Z,14Z)); 1-tridecanoyl- } \\
\text { 2-(8Z,11Z,14Z-eicosatrienoyl)-glycero-3P }\end{array}$ & $\begin{array}{l}\text { PA(15:0/18:3(6Z,9Z,12Z)); 1- } \\
\text { pentadecanoyl-2-(6Z,9Z,12Z- } \\
\text { octadecatrienoyl)-glycero-3P }\end{array}$ \\
\hline 17357 & 0.0202 & -2.710 & $\mathrm{C}_{43} \mathrm{H}_{79} \mathrm{O}_{8} \mathrm{P}$ & Lp & 753.544006 & $\begin{array}{l}\text { PA(18:1(9Z)/22:2(13Z,16Z)); 1-(9Z- } \\
\text { octadecenoyl)-2-(13Z,16Z-docosadienoyl)- } \\
\text { glycero-3P }\end{array}$ & $\begin{array}{l}\text { PA(18:2(9Z,12Z)/22:1(11Z)); 1-(9Z,12Z- } \\
\text { octadecadienoyl)-2-(11Z-docosenoyl)- } \\
\text { glycero-3P }\end{array}$ \\
\hline 17171 & 0.0200 & -2.691 & $\mathrm{C}_{39} \mathrm{H}_{69} \mathrm{O}_{10} \mathrm{P}$ & Lp & 727.455621 & $\begin{array}{l}\text { PG(13:0/20:4(5Z,8Z,11Z,14Z)); 1- } \\
\text { tridecanoyl-2-(5Z,8Z,11Z,14Z- } \\
\text { eicosatetraenoyl)-glycero-3-phospho- } \\
\text { (1'-sn-glycerol) }\end{array}$ & $\begin{array}{l}\text { PG(15:0/18:4(6Z,9Z,12Z,15Z)); 1- } \\
\text { pentadecanoyl-2-(6Z,9Z,12Z,15Z- } \\
\text { octadecatetraenoyl)-glycero-3-phospho- } \\
\left(1^{\prime} \text {-sn-glycerol) }\right.\end{array}$ \\
\hline 12566 & 0.0137 & -2.682 & $\mathrm{C}_{21} \mathrm{H}_{39} \mathrm{O}_{7} \mathrm{P}$ & Lp & 433.236085 & Oleoylglycerone phosphate & $\begin{array}{l}\text { LPA(0:0/18:2(9Z,12Z)) (see KEGG } \\
\text { C00416); 2-(9Z,12Z-octadecadienoyl)- } \\
\text { glycero-3P }\end{array}$ \\
\hline 17364 & 0.0213 & -2.680 & $\mathrm{C}_{43} \mathrm{H}_{81} \mathrm{O}_{8} \mathrm{P}$ & Lp & 755.560008 & $\begin{array}{l}\text { PA(18:0/22:2(13Z,16Z)); 1-octadecanoyl-2- } \\
\quad(13 Z, 16 Z \text {-docosadienoyl)-glycero-3P }\end{array}$ & $\begin{array}{l}\text { PA(18:1(9Z)/22:1(11Z)); 1-(9Z- } \\
\text { octadecenoyl)-2-(11Z-docosenoyl)- } \\
\text { glycero-3P }\end{array}$ \\
\hline 17284 & 0.0259 & -2.663 & $\mathrm{C}_{40} \mathrm{H}_{73} \mathrm{O}_{10} \mathrm{P}$ & $\mathrm{Lp}$ & 743.487155 & $\begin{array}{l}\text { PG(16:0/18:3(6Z,9Z,12Z)); 1-hexadecanoyl- } \\
\text { 2-(6Z,9Z,12Z-octadecatrienoyl)-sn- } \\
\text { glycero-3-phospho-(1'-sn-glycerol) }\end{array}$ & $\begin{array}{l}\text { PG(16:0/18:3(9Z,12Z,15Z)); 1- } \\
\text { hexadecanoyl-2-(9Z,12Z,15Z- } \\
\text { octadeatrienoyl)-sn-glycero-3-phospho- } \\
\text { (1'-sn-glycerol) }\end{array}$ \\
\hline 17301 & 0.0213 & -2.652 & $\mathrm{C}_{40} \mathrm{H}_{75} \mathrm{O}_{10} \mathrm{P}$ & Lp & 745.502826 & $\begin{array}{l}\text { PG(16:0/18:2(9Z,12Z)); 1-hexadecanoyl-2- } \\
\text { (9Z,12Z-octadecadienoyl)-sn-glycero-3- } \\
\text { phospho-(1'-sn-glycerol) }\end{array}$ & $\begin{array}{l}\text { PG(16:1(9Z)/18:1(11Z)); 1-(9Z- } \\
\text { hexadecenoyl)-2-(11Z-octadecenoyl)-sn- } \\
\left.\text { glycero-3-phospho-( } 1^{\prime} \text {-sn-glycerol }\right)\end{array}$ \\
\hline 11978 & 0.0140 & -2.626 & $\mathrm{C}_{19} \mathrm{H}_{39} \mathrm{O}_{7} \mathrm{P}$ & Lp & 409.236062 & $\begin{array}{l}\text { 1-Palmitoylglycerol 3P; 1-hexadecanoyl- } \\
\text { sn-glycero-3P }\end{array}$ & $\begin{array}{l}\text { LPA(0:0/16:0) (see KEGG C00416); 2- } \\
\text { hexadecanoyl-glycero-3P }\end{array}$ \\
\hline 16981 & 0.0219 & -2.625 & $\mathrm{C}_{37} \mathrm{H}_{71} \mathrm{O}_{10} \mathrm{P}$ & $\mathrm{Lp}$ & 705.471462 & $\begin{array}{l}\text { PG(12:0/19:1(9Z)); 1-dodecanoyl-2-(9Z- } \\
\text { nonadecenoyl)-glycero-3-phospho-(1'- } \\
\text { sn-glycerol) }\end{array}$ & $\begin{array}{l}\text { PG(13:0/18:1(9Z)); 1-tridecanoyl-2-(9Z- } \\
\text { octadecenoyl)-glycero-3-phospho-(1' - } \\
\text { sn-glycerol) }\end{array}$ \\
\hline 11190 & 0.0055 & -2.624 & $\mathrm{C}_{21} \mathrm{H}_{16} \mathrm{O}_{7}$ & $\mathrm{Ph}$ & 379.082660 & Diphyllin & Tetracenomycin F1 methylester \\
\hline 17699 & 0.0168 & -2.620 & $\mathrm{C}_{41} \mathrm{H}_{77} \mathrm{O}_{13} \mathrm{P}$ & Lp & 807.503427 & $\begin{array}{l}\text { PI(16:0/16:1(9Z)) (see KEGG C00626); 1- } \\
\text { hexadecanoyl-2-(9Z-hexadecenoyl)-sn- } \\
\text { glycero-3-phosphoinositol }\end{array}$ & $\begin{array}{l}\text { PI(16:1(9Z)/16:0) (see KEGG C00626); } \\
\text { 1-(9Z-hexadecenoyl)-2-hexadecanoyl- } \\
\text { sn-glycero-3-phosphoinositol }\end{array}$ \\
\hline 16538 & 0.0188 & -2.617 & $\mathrm{C}_{37} \mathrm{H}_{67} \mathrm{O}_{8} \mathrm{P}$ & Lp & 669.450309 & $\begin{array}{l}\text { PA(14:0/20:3(8Z,11Z,14Z)); 1- } \\
\text { tetradecanoyl-2-(8Z,11Z,14Z- } \\
\text { eicosatrienoyl)-glycero-3P }\end{array}$ & $\begin{array}{l}\mathrm{PA}(14: 1(9 \mathrm{Z}) / 20: 2(11 \mathrm{Z}, 14 \mathrm{Z})) ; 1-(9 \mathrm{Z}- \\
\text { tetradecenoyl)-2-(11Z,14Z-eicosadienoyl)- } \\
\text { glycero-3P }\end{array}$ \\
\hline 17118 & 0.0280 & -2.614 & $\mathrm{C}_{38} \mathrm{H}_{75} \mathrm{O}_{10} \mathrm{P}$ & $\mathrm{Lp}$ & 721.502885 & $\begin{array}{l}\text { PG(16:0/16:0); 1,2-dihexadecanoyl-sn- } \\
\text { glycero-3-phospho-(1'-sn-glycerol) }\end{array}$ & $\begin{array}{l}\text { PG(18:0/14:0); 1-octadecanoyl-2- } \\
\text { tetradecanoyl-sn-glycero-3-phospho- } \\
\left(1^{\prime} \text {-sn-glycerol }\right)\end{array}$ \\
\hline 16562 & 0.0201 & -2.573 & $\mathrm{C}_{37} \mathrm{H}_{69} \mathrm{O}_{8} \mathrm{P}$ & Lp & 671.466004 & $\begin{array}{l}\text { PA(16:0/18:2(9Z,12Z)) (see KEGG } \\
\text { C00416); 1-hexadecanoyl-2-(9Z,12Z- } \\
\text { octadecadienoyl)-sn-glycero-3P }\end{array}$ & $\begin{array}{l}\text { PA(16:0/18:2(9Z,12Z)); 1-hexadecanoyl-2- } \\
\quad(9 Z, 12 Z \text {-octadecadienoyl)-sn-glycero-3P }\end{array}$ \\
\hline 16702 & 0.0097 & -2.572 & $\mathrm{C}_{38} \mathrm{H}_{67} \mathrm{O}_{8} \mathrm{P}$ & Lp & 681.45028 & $\begin{array}{l}\mathrm{PA}(13: 0 / 22: 4(7 \mathrm{Z}, 10 \mathrm{Z}, 13 \mathrm{Z}, 16 \mathrm{Z})) ; 1- \\
\text { tridecanoyl-2-(7Z,10Z,13Z,16Z- } \\
\text { docosatetraenoyl)-glycero-3P }\end{array}$ & $\begin{array}{l}\text { PA(15:0/20:4(5Z,8Z,11Z,14Z)); 1- } \\
\text { pentadecanoyl-2-(5Z,8Z,11Z,14Z- } \\
\text { eicosatetraenoyl)-glycero-3P }\end{array}$ \\
\hline 15365 & 0.0233 & -2.537 & $\mathrm{C}_{25} \mathrm{H}_{32} \mathrm{O}_{14}$ & $\mathrm{Ph}$ & 555.172014 & 7-Dehydrologanin tetraacetate & Deutzioside pentaacetate \\
\hline
\end{tabular}

a Selection was made using Perseus applying $t$ test $\left(\mathrm{FDR}=0.03\right.$ and $\left.\mathrm{S}_{0}=1\right)$ and components are ranked according to their $t$ test statistic values. If multiple MassTrix annotations for the same formula were obtained, only the two first ones are listed. AC, white wood of asymptomatic vines; AD, white wood of diseased vines; ID, component identity; $P, t$ test associated $P$ value; Cat., multidimensional stoichiometric constraints classification (MSCC) predicted structural category; amino sugars (As); phytochemicals (Ph); peptides (Pp); lipids (Lp); carbohydrates (Cb); and $\mathrm{m} / \mathrm{z}$, measured mass value. 
TABLE 2. (Continued from previous page)

\begin{tabular}{|c|c|c|c|c|c|c|c|}
\hline ID & $P$ & $t$ test & Formula & Cat. & $m / z$ & \multicolumn{2}{|c|}{ Annotations } \\
\hline 16853 & 0.0227 & -2.535 & $\mathrm{C}_{39} \mathrm{H}_{67} \mathrm{O}_{8} \mathrm{P}$ & Lp & 693.450342 & $\begin{array}{l}\mathrm{PA}(14: 1(9 \mathrm{Z}) / 22: 4(7 \mathrm{Z}, 10 \mathrm{Z}, 13 \mathrm{Z}, 16 \mathrm{Z})) ; 1-(9 \mathrm{Z}- \\
\quad \text { tetradecenoyl)-2-(7Z,10Z,13Z,16Z- } \\
\text { docosatetraenoyl)-glycero-3P }\end{array}$ & $\begin{array}{l}\text { PA(18:1(9Z)/18:4(6Z,9Z,12Z,15Z)); } \\
\text { 1-(9Z-octadecenoyl)-2-(6Z,9Z,12Z,15Z- } \\
\text { octadecatetraenoyl)-glycero-3P }\end{array}$ \\
\hline 16436 & 0.0280 & -2.533 & $\mathrm{C}_{36} \mathrm{H}_{67} \mathrm{O}_{8} \mathrm{P}$ & $\mathrm{Lp}$ & 657.450318 & $\begin{array}{l}\text { PA(13:0/20:2(11Z,14Z)); 1-tridecanoyl-2- } \\
\quad(11 Z, 14 Z \text {-eicosadienoyl)-glycero-3P }\end{array}$ & $\begin{array}{l}\text { PA }(14: 1(9 Z) / 19: 1(9 Z)) ; 1-(9 Z- \\
\text { tetradecenoyl)-2-(9Z-nonadecenoyl)- } \\
\text { glycero-3P }\end{array}$ \\
\hline 17113 & 0.0046 & -2.524 & $\mathrm{C}_{33} \mathrm{H}_{38} \mathrm{O}_{18}$ & $\mathrm{Ph}$ & 721.199196 & 6"'-(3-Hydroxy-3-methylglutaroyl)isoviolanthin & \\
\hline 16871 & 0.0241 & -2.523 & $\mathrm{C}_{39} \mathrm{H}_{69} \mathrm{O}_{8} \mathrm{P}$ & Lp & 695.466015 & $\begin{array}{l}\text { PA(16:0/20:4(5Z,8Z,11Z,14Z)); 1- } \\
\text { hexadecanoyl-2-(5Z,8Z,11Z,14Z- } \\
\text { eicosatetraenoyl)-sn-glycero-3P }\end{array}$ & $\begin{array}{l}\text { PA(14:0/22:4(7Z,10Z,13Z,16Z)); 1- } \\
\quad \text { tetradecanoyl-2-(7Z,10Z,13Z,16Z- } \\
\text { docosatetraenoyl)-glycero-3P }\end{array}$ \\
\hline 16337 & 0.0177 & -2.516 & $\mathrm{C}_{35} \mathrm{H}_{69} \mathrm{O}_{8} \mathrm{P}$ & $\mathrm{Lp}$ & 647.465942 & $\begin{array}{l}\text { PA(16:0/16:0) (see KEGG C00416); 1,2- } \\
\text { dihexadecanoyl-rac-glycero-3P }\end{array}$ & $\begin{array}{l}\text { PA(16:0/16:0)[rac]; 1,2-dihexadecanoyl- } \\
\text { sn-glycero-3P }\end{array}$ \\
\hline 16924 & 0.0289 & -2.505 & $\mathrm{C}_{39} \mathrm{H}_{73} \mathrm{O}_{8} \mathrm{P}$ & Lp & 699.497294 & $\begin{array}{l}\text { PA(18:0/18:2(9Z,12Z)) (see KEGG } \\
\text { C00416); 1-octadecanoyl-2-(9Z, } \\
\text { 12Z-octadecadienoyl)-sn-glycero-3P }\end{array}$ & $\begin{array}{l}\text { PA(18:1(11Z)/18:1(11Z)) (see KEGG } \\
\text { C00416); 1,2-di(11Z-octadecenoyl)- } \\
\text { rac-glycero-3P }\end{array}$ \\
\hline 15250 & 0.0086 & -2.503 & $\mathrm{C}_{28} \mathrm{H}_{55} \mathrm{O}_{8} \mathrm{P}$ & Lp & 549.35616 & PA(13:0/12:0); 1-tridecanoyl-2-dodecanoyl-glyc & cero-3P \\
\hline 16184 & 0.0092 & -2.481 & $\mathrm{C}_{34} \mathrm{H}_{65} \mathrm{O}_{8} \mathrm{P}$ & $\mathrm{Lp}$ & 631.434712 & $\begin{array}{l}\text { PA(12:0/19:1(9Z)); 1-dodecanoyl-2-(9Z- } \\
\text { nonadecenoyl)-glycero-3P }\end{array}$ & $\begin{array}{l}\text { PA(13:0/18:1(9Z)); 1-tridecanoyl-2-(9Z- } \\
\text { octadecenoyl)-glycero-3P }\end{array}$ \\
\hline 13509 & 0.0092 & -2.480 & $\mathrm{C}_{21} \mathrm{H}_{39} \mathrm{O}_{9} \mathrm{P}$ & $\mathrm{Lp}$ & 465.225763 & $\begin{array}{l}\text { Dolichyl b-D-glucosyl phosphate (see KEGG CC } \\
\text { oxan-2-yl]oxy-(3,7,11-trimethyldodeca- } 6,10 \text {-d }\end{array}$ & $\begin{array}{l}\text { 01246); [3,4,5-trihydroxy-6-(hydroxymethyl) } \\
\text { lienoxy)phosphinic acid }\end{array}$ \\
\hline 17172 & 0.0276 & -2.470 & $\mathrm{C}_{41} \mathrm{H}_{77} \mathrm{O}_{8} \mathrm{P}$ & $\mathrm{Lp}$ & 727.528589 & $\begin{array}{l}\text { PA(16:0/22:2(13Z,16Z)); 1-hexadecanoyl-2- } \\
\quad(13 Z, 16 Z \text {-docosadienoyl)-glycero-3P }\end{array}$ & $\begin{array}{l}\text { PA }(16: 1(9 Z) / 22: 1(11 Z)) ; 1-(9 Z- \\
\text { hexadecenoyl)-2-(11Z-docosenoyl)- } \\
\text { glycero-3P }\end{array}$ \\
\hline 12745 & 0.0225 & -2.463 & $\mathrm{C}_{20} \mathrm{H}_{24} \mathrm{O}_{9} \mathrm{~S}$ & $\mathrm{Ph}$ & 439.106482 & Hallactone B & $\begin{array}{l}\mathrm{R} \text { replaced by } \mathrm{H} \text { in } 3,5,7,9,11,13,15,17- \\
\text { octaoxo-eicosanoyl-[acp] }\end{array}$ \\
\hline 6773 & 0.0115 & +3.849 & $\mathrm{C}_{10} \mathrm{H}_{19} \mathrm{NO}_{4}$ & $\mathrm{Lp}$ & 216.124136 & O-Propanoylcarnitine; O-propionylcarnitine & $\begin{array}{l}\text { Propionylcarnitine (see KEGG C03017); } \\
\text { 3-propanoyloxy-4-trimethylammonio- } \\
\text { butanoate }\end{array}$ \\
\hline
\end{tabular}

TABLE 3. List of the 24 top annotated $m / z$ accumulated in Chardonnay SD samples compared with AC samples ${ }^{\mathrm{a}}$

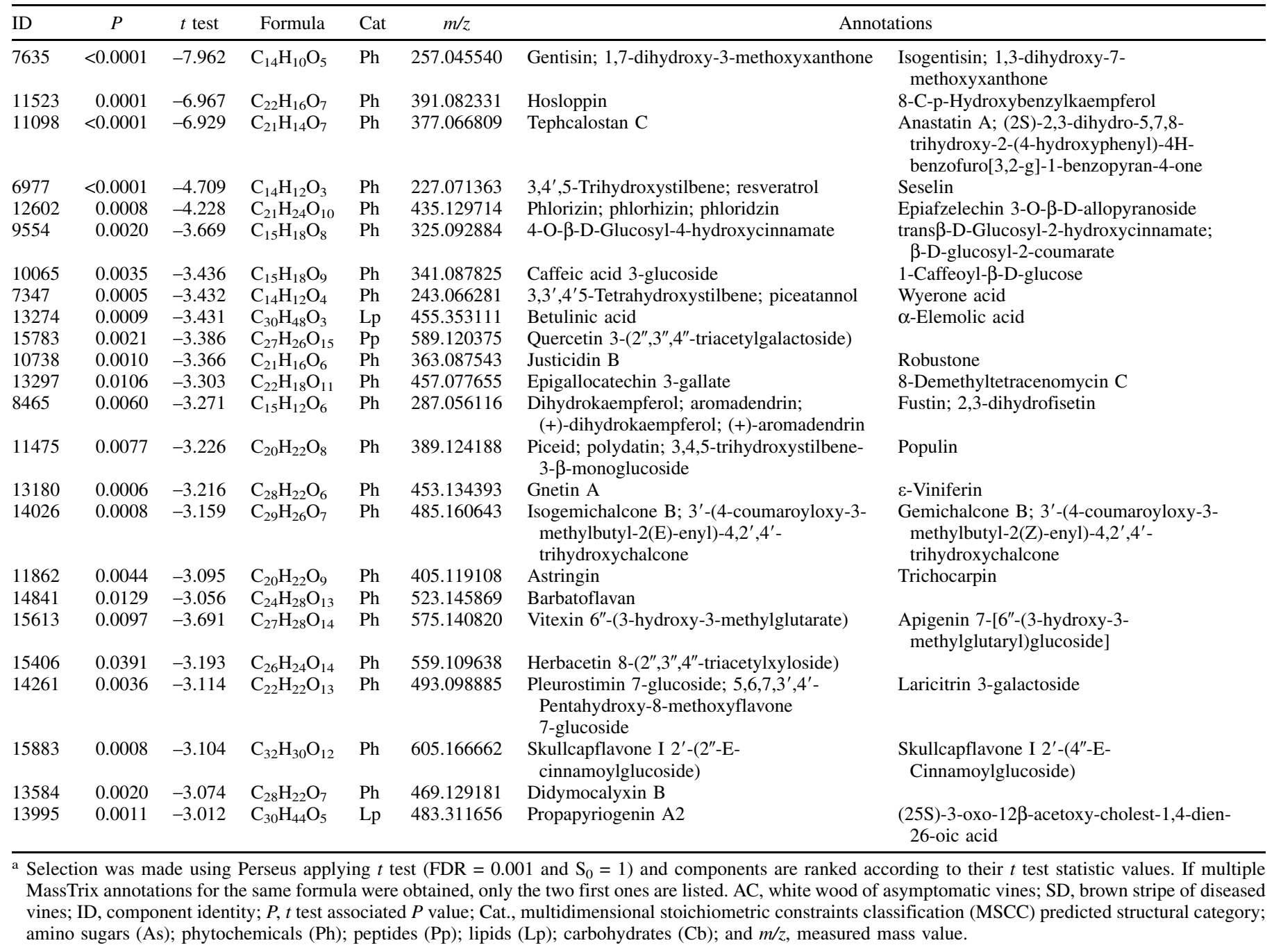


discriminant compounds ("top compounds") were determined in Perseus by strengthening Student's $t$ test parameters such as permutation-based false discovery rate (FDR) and $\mathrm{S}_{0}$ (two groups threshold fold change). (Supplementary Fig. S2, step f). The resulting significant outliers (FDR and $\mathrm{S}_{0}$ values detailed on related Tables) were depicted among the identified compounds.

\section{RESULTS}

Whole dataset information. The FT-ICR-MS analysis of all provided a total of $6,499 \mathrm{~m} / \mathrm{z}$ that were reduced to 5,842 with validated elemental formulas. These 5,842 formulas covered a high chemical diversity, as shown by the van Krevelen diagram, which represents their distribution on the basis of their $\mathrm{H} / \mathrm{C}$ and $\mathrm{O} / \mathrm{C}$ ratios (Supplementary Fig. S3A). Higher proportions of $\mathrm{CHO}, \mathrm{CHON}$, and CHONS species $(37,22$, and $15 \%$, respectively) were observed (Supplementary Fig. S3A). These 5,842 formulas were principally equally attributed to phytochemicals and lipids (27 and $28 \%$, respectively), peptides (22\%), and then nonmatching (NM) structures (15\%) (Supplementary Fig. S3B). For further statistical analyses, data curation based on replicate redundancy was applied on the whole data set, which led to $6,277 \mathrm{~m} / \mathrm{z}$.

Statistical analysis (ANOVA, $P$ value $<0.05$ ) was then performed on the $6,277 \mathrm{~m} / \mathrm{z}$. for [cultivar_wood area] discrimination. This analysis highlighted 3,890 significant $\mathrm{m} / \mathrm{z}$ values. However, the global HCA did not allow a clear discrimination of groups based on cultivars and/or disease (Supplementary Fig. S4). We therefore performed data analysis by two ways. First, the whole data set was split into three subsets according to the cultivar (one by cultivar), in order to get their $\mathrm{AC}, \mathrm{AD}$, and $\mathrm{SD}$ metabolic fingerprints and

TABLE 4. List of the 33 top $m / z$ accumulated in Gewurztraminer SD samples compared with AC samples ${ }^{\mathrm{a}}$

\begin{tabular}{|c|c|c|c|c|c|c|c|}
\hline ID & $P$ & $t$ test & Formula & Cat. & $m / z$ & Anno & ations \\
\hline 8690 & $<0.0001$ & -5.032 & $\mathrm{C}_{17} \mathrm{H}_{12} \mathrm{O}_{5}$ & $\mathrm{Ph}$ & 295.061199 & Aspulvinone $\mathrm{E}$ & $\begin{array}{l}\text { Pseudobaptigenin methyl ether; 7-methoxy- } \\
3^{\prime}, 4^{\prime} \text {-methylenedioxyisoflavone }\end{array}$ \\
\hline 8121 & $<0.0001$ & -4.548 & $\mathrm{C}_{15} \mathrm{H}_{14} \mathrm{O}_{5}$ & $\mathrm{Ph}$ & 273.076861 & Phloretin & $\alpha$-Pyrufuran \\
\hline 12161 & 0.0003 & -4.167 & $\mathrm{C}_{21} \mathrm{H}_{24} \mathrm{O}_{9}$ & $\mathrm{Ph}$ & 419.134766 & Melampodin A & Glycyphyllin; phloretin 2'-O-rhamnoside \\
\hline 11533 & $<0.0001$ & -4.104 & $\mathrm{C}_{20} \mathrm{H}_{24} \mathrm{O}_{8}$ & $\mathrm{Ph}$ & 391.139864 & Eupatoroxin & 10-epi-Eupatoroxin \\
\hline 6697 & $<0.0001$ & -4.040 & $\mathrm{C}_{14} \mathrm{H}_{12} \mathrm{O}_{2}$ & $\mathrm{Ph}$ & 211.076478 & $\begin{array}{l}\text { Benzoin; 2-hydroxy-1,2-diphenylethanone; } \\
\text { benzoin tincture }\end{array}$ & Pinosylvin \\
\hline 9308 & $<0.0001$ & -4.034 & $\mathrm{C}_{17} \mathrm{H}_{16} \mathrm{O}_{6}$ & $\mathrm{Ph}$ & 315.087404 & Cajanol & Homoferreirin \\
\hline 12369 & $<0.0001$ & -3.980 & $\mathrm{C}_{20} \mathrm{H}_{25} \mathrm{ClO}_{8}$ & $\mathrm{Ph}$ & 427.116559 & Eupachloroxin & \\
\hline 12549 & $<0.0001$ & -3.968 & $\mathrm{C}_{21} \mathrm{H}_{22} \mathrm{O}_{10}$ & $\mathrm{Ph}$ & 433.114055 & Naringenin 7-O- $\beta$-D-glucoside; prunin & Phlorizin chalcone; chalcone $2^{\prime}$-O-glucoside \\
\hline 14026 & $<0.0001$ & -3.934 & $\mathrm{C}_{29} \mathrm{H}_{26} \mathrm{O}_{7}$ & $\mathrm{Ph}$ & 485.160643 & $\begin{array}{l}\text { Isogemichalcone B; 3'-(4-coumaroyloxy-3- } \\
\text { methylbutyl-2(E)-enyl)-4,2', } 4^{\prime}- \\
\text { trihydroxychalcone }\end{array}$ & $\begin{array}{l}\text { Gemichalcone B; 3'-(4-coumaroyloxy-3- } \\
\text { methylbutyl-2(Z)-enyl)-4,2', } 4^{\prime}- \\
\text { trihydroxychalcone }\end{array}$ \\
\hline 10782 & $<0.0001$ & -3.906 & $\mathrm{C}_{13} \mathrm{H}_{19} \mathrm{O}_{10} \mathrm{P}$ & $\mathrm{Ph}$ & 365.064466 & Salicin $6 \mathrm{P}$ & \\
\hline 10675 & 0.0012 & -3.902 & $\mathrm{C}_{16} \mathrm{H}_{15} \mathrm{~N}_{2} \mathrm{O}_{6} \mathrm{P}$ & $\mathrm{Ph}$ & 361.059764 & Fosphenytoin & \\
\hline 9223 & $<0.0001$ & -3.898 & $\mathrm{C}_{17} \mathrm{H}_{14} \mathrm{O}_{6}$ & $\mathrm{Ph}$ & 313.071873 & Ventinone A & Irisolidone \\
\hline 9783 & 0.0002 & -3.793 & $\mathrm{C}_{17} \mathrm{H}_{16} \mathrm{O}_{7}$ & $\mathrm{Ph}$ & 331.082093 & Sulochrin & $3^{\prime}$-Deoxydryopteric acid \\
\hline 9272 & 0.0001 & -3.741 & $\mathrm{C}_{16} \mathrm{H}_{12} \mathrm{O}_{7}$ & $\mathrm{Ph}$ & 315.050985 & $\begin{array}{l}\text { 3', } 4^{\prime}, 5,7-\text { Tetrahydroxy-3-methoxyflavone; } \\
\text { 3-methoxy-5,7,3', } 4^{\prime} \text {-tetrahydroxyflavone; } \\
\text { 3-O-methylquercetin }\end{array}$ & Capillarisin \\
\hline 6004 & 0.0019 & -3.722 & $\mathrm{C}_{6} \mathrm{H}_{10} \mathrm{O}_{5}$ & $\mathrm{Cb}$ & 161.045569 & Lichenin; lichenan & (R)-3,3-Dimethylmalate \\
\hline 15851 & 0.0001 & -3.692 & $\mathrm{C}_{27} \mathrm{H}_{32} \mathrm{O}_{15}$ & $\mathrm{Ph}$ & 595.167016 & Isobutrin & Butrin; butin 7,3'-di-O-glucoside \\
\hline 10621 & 0.0001 & -3.691 & $\mathrm{C}_{22} \mathrm{H}_{16} \mathrm{O}_{5}$ & $\mathrm{Ph}$ & 359.092546 & Pinocembrin 7-O-benzoate & \\
\hline 12189 & 0.0016 & -3.657 & $\mathrm{C}_{20} \mathrm{H}_{22} \mathrm{O}_{10}$ & $\mathrm{Ph}$ & 421.114102 & Catechin 7-O- $\beta$-D-xyloside & Plicatic acid \\
\hline 8017 & 0.0001 & -3.625 & $\mathrm{C}_{16} \mathrm{H}_{14} \mathrm{O}_{4}$ & $\mathrm{Ph}$ & 269.081951 & $\begin{array}{l}\text { (+)-Medicarpin; 3-hydroxy-9- } \\
\text { methoxypterocarpan }\end{array}$ & Vignafuran \\
\hline 13108 & 0.0016 & -3.614 & $\mathrm{C}_{21} \mathrm{H}_{24} \mathrm{O}_{11}$ & $\mathrm{Ph}$ & 451.124596 & 6-C-Glucopyranosylepicatechin & 8-C-Glucopyranosylepicatechin \\
\hline 12556 & 0.0001 & -3.594 & $\mathrm{C}_{22} \mathrm{H}_{26} \mathrm{O}_{9}$ & $\mathrm{Ph}$ & 433.150351 & Eleganin & Graminiliatrin \\
\hline 13052 & 0.0007 & -3.570 & $\mathrm{C}_{21} \mathrm{H}_{22} \mathrm{O}_{11}$ & $\mathrm{Ph}$ & 449.108960 & Neoastilbin; (2S,3S)-taxifolin 3-rhamnoside & $\begin{array}{l}2^{\prime}, 3,4,4^{\prime}, 6^{\prime} \text {-Peptahydroxychalcone } 4^{\prime} \text {-O- } \\
\text { glucoside; PHC } 4^{\prime} \text {-O-glucoside }\end{array}$ \\
\hline 12602 & 0.0007 & -3.569 & $\mathrm{C}_{21} \mathrm{H}_{24} \mathrm{O}_{10}$ & $\mathrm{Ph}$ & 435.129714 & Phlorizin; phlorhizin; phloridzin & Epiafzelechin 3-O- $\beta$-D-allopyranoside \\
\hline 8941 & 0.0006 & -3.558 & $\mathrm{C}_{15} \mathrm{H}_{12} \mathrm{O}_{7}$ & $\mathrm{Ph}$ & 303.051030 & $\begin{array}{l}\text { Taxifolin; dihydroquercetin; } \\
\text { (+)-dihydroquercetin; (+)-taxifolin; trans- } \\
\text { dihydroquercetin }\end{array}$ & Pentahydroxyflavanone; dihydrotricetin \\
\hline 13104 & 0.0020 & -3.546 & $\mathrm{C}_{24} \mathrm{H}_{20} \mathrm{O}_{9}$ & $\mathrm{Ph}$ & 451.103486 & Cinchonain 1a & $\begin{array}{l}\text { 9,10-Dihydro-10-(3,4-dihydroxyphenyl)- } \\
\text { pyrano[2,3-h]catechin-8-one }\end{array}$ \\
\hline 8748 & 0.0002 & -3.510 & $\mathrm{C}_{17} \mathrm{H}_{14} \mathrm{O}_{5}$ & $\mathrm{Ph}$ & 297.076935 & $\begin{array}{l}\text { Apigenin } 7,4^{\prime} \text {-dimethyl ether; apigenin } \\
\text { dimethylether }\end{array}$ & Afrormosin \\
\hline 13497 & 0.0009 & -3.506 & $\mathrm{C}_{21} \mathrm{H}_{22} \mathrm{O}_{12}$ & $\mathrm{Ph}$ & 465.103886 & Quercetin 7-O-glucoside & Plantagoside \\
\hline 10401 & 0.0002 & -3.483 & $\mathrm{C}_{21} \mathrm{H}_{18} \mathrm{O}_{5}$ & $\mathrm{Ph}$ & 349.108165 & Maximaisoflavone B & 4'-O-methylalpinumisoflavone \\
\hline 12598 & 0.0002 & -3.482 & $\mathrm{C}_{24} \mathrm{H}_{20} \mathrm{O}_{8}$ & $\mathrm{Ph}$ & 435.108547 & $\begin{array}{l}\text { 9,10-Dihydro-10-(4-hydroxyphenyl)-pyrano } \\
\text { [2,3-h]epicatechin-8-one }\end{array}$ & Epigallocatechin 3-O-cinnamate \\
\hline 10350 & 0.0002 & -3.462 & $\mathrm{C}_{21} \mathrm{H}_{16} \mathrm{O}_{5}$ & $\mathrm{Ph}$ & 347.092536 & $\begin{array}{l}\text { Calopogonium isoflavone B; } 6^{\prime \prime}, 6^{\prime \prime} \text {-dimethyl- } \\
3^{\prime}, 4^{\prime} \text {-methylenedioxypyrano }\left[2^{\prime \prime}, 3^{\prime \prime}: 7,8\right] \\
\text { isoflavone }\end{array}$ & Tuberostan \\
\hline 6555 & 0.0002 & -3.444 & $\mathrm{C}_{13} \mathrm{H}_{14} \mathrm{O}_{2}$ & $\mathrm{Ph}$ & 201.092122 & Tremetone & \\
\hline 8424 & 0.0002 & -3.414 & $\mathrm{C}_{13} \mathrm{H}_{18} \mathrm{O}_{7}$ & $\mathrm{Ph}$ & 285.097974 & Salicin; salicoside & $\begin{array}{l}\text { Gastrodin; 4-(hydroxymethyl)phenyl- } \beta \text {-D- } \\
\text { glucopyranoside }\end{array}$ \\
\hline 6805 & 0.0008 & -3.252 & $\mathrm{C}_{9} \mathrm{H}_{17} \mathrm{NO}_{5}$ & Lp & 218.103393 & $\begin{array}{l}\text { Pantothenate; pantothenic acid; (R)- } \\
\text { pantothenate }\end{array}$ & $\begin{array}{l}\text { Pantothenic acid (see KEGG C00864); } \\
\text { 3-[[(2R)-2,4-dihydroxy-3,3-dimethyl- } \\
\text { butanoyl]amino]propanoic acid }\end{array}$ \\
\hline
\end{tabular}

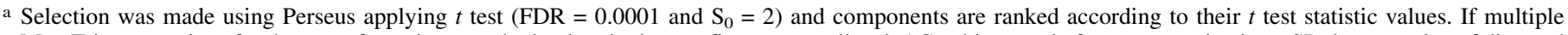
MassTrix annotations for the same formula were obtained, only the two first ones are listed. AC, white wood of asymptomatic vines; SD, brown stripe of diseased vines; ID, component identity; $P, t$ test associated $P$ value; Cat., multidimensional stoichiometric constraints classification (MSCC) predicted structural category; amino sugars (As); phytochemicals $(\mathrm{Ph})$; peptides $(\mathrm{Pp})$; lipids (Lp); carbohydrates $(\mathrm{Cb})$; and $\mathrm{m} / z$, measured mass value. 
TABLE 5. List of the 50 top $\mathrm{m} / z$ differentially accumulated in Mourvèdre AC and SD samples ${ }^{\mathrm{a}}$

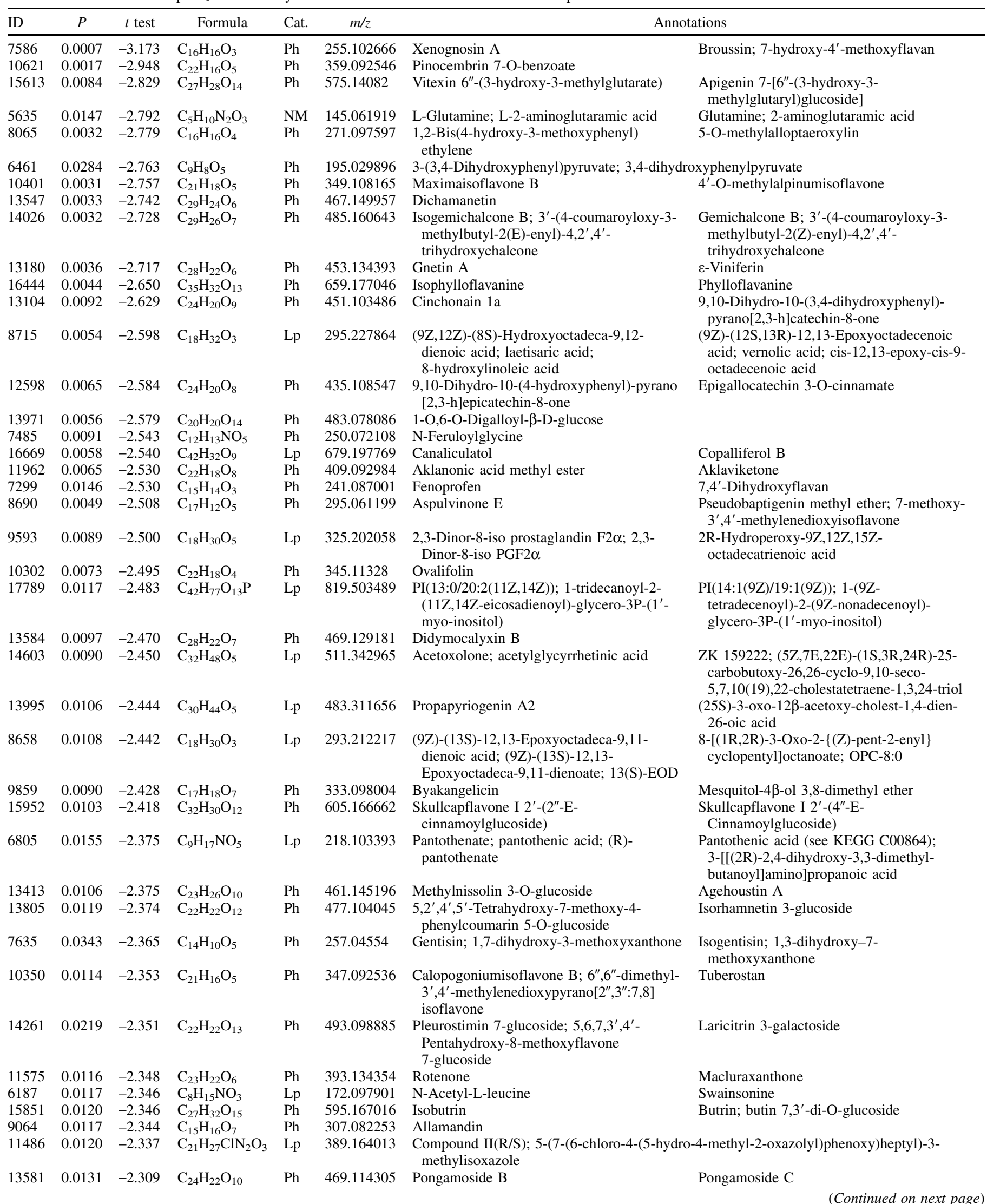

a $\mathrm{m} / \mathrm{z}$ are either accumulated in AC (positive $t$ statistic value) or in SD (negative $t$ statistic value). Selection was made using Perseus applying $t$ test (FDR $=0.04$ and $\mathrm{S}_{0}=2$ ) and components are ranked according to their $\mathrm{t}$ test statistic values. If multiple MassTrix annotations for the same formula were obtained, only the two first ones are listed. AC, white wood of asymptomatic vines; SD, brown stripe of diseased vines; ID, component identity; $P, t$ test associated $P$ value; Cat., multidimensional stoichiometric constraints classification (MSCC) predicted structural category; amino sugars (As); phytochemicals (Ph); peptides (Pp); lipids (Lp); carbohydrates $(\mathrm{Cb})$; and $\mathrm{m} / \mathrm{z}$, measured mass value. 
TABLE 5. (Continued from previous page)

\begin{tabular}{|c|c|c|c|c|c|c|c|}
\hline ID & $P$ & $t$ test & Formula & Cat. & $m / z$ & Annota & ations \\
\hline 14573 & 0.0227 & -2.300 & $\mathrm{C}_{32} \mathrm{H}_{46} \mathrm{O}_{5}$ & Lp & 509.32727 & $\begin{array}{l}\text { 11-(3-acetoxy-1-propynyl)-1 } \alpha, 25 \text { - } \\
\text { dihydroxy-9,11-didehydrovitamin D3 / 11- } \\
\text { (3-acetoxy-1-propynyl)-1 } \alpha, 25 \text {-dihydroxy- } \\
\text { 9,11-didehydrocholecalciferol; (5Z,7E)- } \\
\text { (1S,3R)-11-(3-acetoxy-1-propynyl)-9,10- } \\
\text { seco-5,7,9(11),10(19)-cholestatetraene- } \\
\text { 1,3,25-triol }\end{array}$ & $\begin{array}{l}\text { ZK 168281; (5Z,7E,22E)-(1S,3R,24R)-25- } \\
\text { (carboethoxy-methylene)-26,26-cyclo- } \\
\text { 9,10-seco-5,7,10(19),22-cholestatetraene- } \\
\text { 1,3,24-triol }\end{array}$ \\
\hline 10793 & 0.0139 & -2.289 & $\mathrm{C}_{21} \mathrm{H}_{18} \mathrm{O}_{6}$ & $\mathrm{Ph}$ & 365.103108 & Glycyrol; neoglycyrol & $\begin{array}{l}\text { Derrubone; 5,7-Dihydroxy- } 3^{\prime}, 4^{\prime}- \\
\text { methylenedioxy-6-prenylisoflavone }\end{array}$ \\
\hline 10476 & 0.0155 & -2.254 & $\mathrm{C}_{20} \mathrm{H}_{18} \mathrm{O}_{6}$ & $\mathrm{Ph}$ & 353.103165 & Cyclokievitone & Licoisoflavone A \\
\hline 15158 & 0.0157 & -2.250 & $\mathrm{C}_{27} \mathrm{H}_{28} \mathrm{O}_{12}$ & $\mathrm{Ph}$ & 543.151233 & Premithramycin A1 & \\
\hline 11571 & 0.0157 & -2.250 & $\mathrm{C}_{22} \mathrm{H}_{18} \mathrm{O}_{7}$ & $\mathrm{Ph}$ & 393.098044 & Justicidin A & 12a-Hydroxyisomillettone \\
\hline 13537 & 0.0164 & -2.236 & $\mathrm{C}_{24} \mathrm{H}_{20} \mathrm{O}_{10}$ & $\mathrm{Ph}$ & 467.0984 & Epigallocatechin 3-O-caffeate & Apocynin A \\
\hline 15570 & 0.0167 & -2.229 & $\mathrm{C}_{25} \mathrm{H}_{49} \mathrm{O}_{12} \mathrm{P}$ & $\mathrm{Lp}$ & 571.288944 & $\begin{array}{l}\text { PI(16:0/0:0); 1-hexadecanoyl-sn-glycero-3P- } \\
\text { (1'-myo-inositol) }\end{array}$ & \\
\hline 17113 & 0.0251 & +2.183 & $\mathrm{C}_{33} \mathrm{H}_{38} \mathrm{O}_{18}$ & $\mathrm{Ph}$ & 721.199197 & 6"'-(3-Hydroxy-3-methylglutaroyl)isoviolanthin & \\
\hline 13904 & 0.0172 & +2.392 & $\mathrm{C}_{30} \mathrm{H}_{24} \mathrm{O}_{6}$ & $\mathrm{Lp}$ & 479.150345 & Blestriarene B & \\
\hline
\end{tabular}

compare them. Secondly, $m / z$ values common to the three cultivars were used to identify metabolic fingerprints of $\mathrm{AC}, \mathrm{AD}$, and $\mathrm{SD}$ samples, compare them, and find possible markers.

Cultivar-dependent metabolic fingerprints. Data subsets obtained for Chardonnay, Gewurztraminer, and Mourvèdre were composed of a similar number of $\mathrm{m} / \mathrm{z}$ values among with 3,914, 4,048 , and 3,896, respectively, were assigned with a validated formula (Fig. 1). For each cultivar, lipids and phytochemicals were the two main categories observed at around 30\% (Fig. 1), as observed for the analysis of the global data set (Supplementary Fig. S3B). When compared, the three cultivars shared 2,612 formulas, which were obtained from 2,910 common $\mathrm{m} / \mathrm{z}$ (Fig. 2, top panel). Conversely, 580, 344, and 640 ones were specifically observed in Chardonnay, Gewurztraminer, and Mourvèdre, respectively (Fig. 2, top panel). The 2,612 common formulas were mainly distributed among lipids (32\%) and phytochemicals (25\%) (Fig. 2, bottom panel).

Using ANOVA statistical analysis $(P<0.05)$, significant $m / z$ lists were obtained with a similar number of $\mathrm{m} / \mathrm{z}$, for Chardonnay and Gewurztraminer $(2,171$ and $2,140 \mathrm{~m} / \mathrm{z}$, respectively) and a lower one for Mourvèdre (only $656 \mathrm{~m} / \mathrm{z}$ ) (Supplementary File S1). For each cultivar, the data subset was then $t$ test pair-compared (FDR $<0.05)$ on the basis of sample type (AC, AD, and SD). Significant $\mathrm{m} / \mathrm{z}$ having a reliable elemental formula (determined by VK criteria) were submitted to MassTrix identification. Among them, only annotated $\mathrm{m} / \mathrm{z}$, were finally considered to select the discriminating ones (i.e., the most discriminant putative metabolites) by adjusting both FDR values and $S_{0}$ in Perseus software, in order to get the lowest number of putative discriminant candidates.

For $\mathrm{AC}$ and $\mathrm{AD}$ sample comparison, the greatest number of significant $\mathrm{m} / \mathrm{z}$ differently accumulated was observed for Chardonnay $(1,723 \mathrm{~m} / \mathrm{z})$, then Gewurztraminer $(229 \mathrm{~m} / \mathrm{z})$, whereas only one could be found for Mourvèdre (Fig. 3). Among them, 23 top $\mathrm{m} / z$ were listed for Chardonnay (all accumulated in AD), 39 for Gewurztraminer (38/39 accumulated in $\mathrm{AD}$ ), and none for Mourvèdre (the single discriminant $\mathrm{m} / \mathrm{z}$ was not annotated) (Tables 1 and 2). In both Chardonnay and Gewurztraminer cultivars, all/most of the top metabolites were more accumulated in AD, and were mostly belonging to lipids, mainly fatty acids for Chardonnay and phospholipids (phosphatidic acids, phosphatidyl glycerol, and phosphatidyl inositol) for Gewurztraminer (Tables 1 and 2). Phytochemicals were also accumulated, such as hosloppin, buchananine, and sinapyl alcohol in Chardonnay; diphyllin and 1caffeoyl-4-deoxyquinic acid in Gewurztraminer, but to a lesser extent. Peptides (such as Tryptophan for Chardonnay) and amino sugars (such as $\mathrm{N}$-acetyl-L-glutamate for Gewurztraminer) were scarcely listed.
A similar comparison was made between AC and SD samples. The highest number of $\mathrm{m} / \mathrm{z}$ differently accumulated was observed for Gewurztraminer $(1,701 \mathrm{~m} / \mathrm{z})$, then Mourvèdre $(540 \mathrm{~m} / \mathrm{z})$, and the lowest one for Chardonnay (64 m/z) (Fig. 3). Among them, most were accumulated in SD, 24 top $\mathrm{m} / \mathrm{z}$ were identified for Chardonnay, 33 for Gewurztraminer, and 50 (48/50 accumulated in SD) for Mourvèdre (Tables 3, 4, and 5). For the three cultivars, the metabolites more accumulated in SD samples were mainly phytochemicals (over 87\%). The grapevine stilbene phytoalexins resveratrol, piceid, astringin, and $\varepsilon$-viniferin were among the most accumulated in Chardonnay, while only $\varepsilon$-viniferin was listed in Mourvèdre top ones, and none of them for Gewurztraminer (Tables 3, 4, and 5). Among other phytochemicals accumulated in Gewurztraminer AD samples were phenolic compounds, mainly flavonoids. As examples, one could find quercetin 7-O-glucoside, dihydroquercetin, or naringenin 7-O-beta-D-glucoside, as putative structures (Table 4).

A last discrimination was made between AD and SD samples. The highest number of $\mathrm{m} / \mathrm{z}$ differently accumulated was observed for Chardonnay $(1,628 \mathrm{~m} / \mathrm{z})$, then Gewurztraminer $(1,382 \mathrm{~m} / \mathrm{z})$, whereas none was found for Mourvèdre (Fig. 3). Among them, 28 top $m / z$ were identified for Chardonnay ( $2 / 28$ accumulated in SD, $26 / 28$ accumulated in AD), and 20 for Gewurztraminer (all accumulated in SD) (Tables 6 and 7). For Chardonnay, only the two phytoalexins resveratrol and $\varepsilon$-viniferin were more accumulated in SD areas compared with $\mathrm{AD}$ ones, whereas mostly lipids (fatty acids and phospholipids) were accumulated in AD (57\%) (Table 6). For Gewurztraminer, the top metabolites were mostly phytochemicals among which the two phytoalexins piceid and astringin, flavonoids (i.e., naringenin 7-O-beta-D-glucoside), and other ones of various chemical families (Table 7).

Altogether, these three comparisons pointed out that in diseased vines, phytochemicals largely contribute to the fingerprint of the brown stripe area (SD), whereas lipids contribute to that of the adjacent white area (AD).

Sample type metabolic fingerprints. Data analysis thus allowed a discrimination of the different sample types (AC, AD, and SD) for each cultivar. Further investigation was next conducted in order to highlight the $\mathrm{m} / \mathrm{z}$ characteristic of the three trunk areas, regardless of the cultivar, i.e., putative markers of the three sample types. The $2,910 \mathrm{~m} / \mathrm{z}$ common to the three cultivars (see above and Supplementary File 1) were selected and ANOVA statistical process $(P<0.05)$ was performed to compare sample types.

AC versus AD comparison revealed 139 common $m / z$ values (122 ones with assigned formulas, 68 annotations) between Chardonnay and Gewurztraminer, and none in common with Mourvèdre (Fig. 3, left side). Among the 122 common formulas, $55 \%$ belong to lipids (Fig. 4A). One hundred and fifteen were more abundant in AD $(57 \%$ 
Lipids) for both cultivars, whereas seven were inversely accumulated in $\mathrm{AC}$ and $\mathrm{AD}$ samples, depending on the cultivar (Fig. 4A, noted "conflict"). Among these $115 \mathrm{AD}$-accumulated $\mathrm{m} / z, 68$ could also be annotated by Mass Trix (including 18 isotopologues) (Fig. 3, Table 8). Most of them were lipids including phosphatidic acids (PA), phosphatidylglycerols (PG), and phosphatidyl inositols (PI). A lower number of phytochemicals were accumulated, such as the flavonoid hosloppin. Besides, the three metabolites L-tryptophan, O-propanoylcarnitine, and gratiogenin were inversely accumulated between both cultivars, which renders them ineligible as AD area markers.

For AC versus SD comparison, $33 \mathrm{~m} / \mathrm{z}$ values were common to the three cultivars ( 30 ones with assigned formula, 12 annotations) (Fig. 3 , top of the middle part). Among the 30 common formulas, $70 \%$ belong to phytochemicals. All were more abundant in SD, mainly phytochemicals (70\%) (Fig. 4B). Only $12 \mathrm{~m} / z$ could be annotated by MassTrix, among which two isotopologues (Fig. 3, Table 9), including the grapevine phytoalexins resveratrol, $\varepsilon$-viniferin (isomer of gnetin A), and piceatannol.

A last comparison was made between AD and SD samples (Fig. 3, right side); $235 \mathrm{~m} / z$ (213 with assigned formulas, 63 annotations) were common for Chardonnay and Gewurztraminer but not for Mourvèdre. Among the 213 common formulas, $36 \%$ correspond to phytochemicals and $24 \%$ to lipids (Fig. 4C). Moreover, from them, 94 were more abundant in SD samples, 96 more abundant in AD samples, and 23 showed variable abundance among sample types, depending on the cultivar. Among the 190 formulas similarly accumulated in both cultivars, 57 could be annotated, among which 18 isotopologues (Table 10). When looking at structural categories (Fig. 4C), mainly phytochemicals $(67 \%)$ were accumulated in SD

TABLE 6. List of the 28 top $m / z$ differentially accumulated in Chardonnay AD and SD samples ${ }^{\text {a }}$

\begin{tabular}{|c|c|c|c|c|c|c|c|}
\hline \multirow{2}{*}{$\frac{\text { ID }}{6977}$} & \multirow{2}{*}{$\frac{P}{<0.0001}$} & \multirow{2}{*}{$\frac{t \text { test }}{-4.120}$} & \multirow{2}{*}{$\frac{\text { Formula }}{\mathrm{C}_{14} \mathrm{H}_{12} \mathrm{O}_{3}}$} & \multirow{2}{*}{$\frac{\text { Cat. }}{\mathrm{Ph}}$} & \multirow{2}{*}{$\frac{m / z}{227.071363}$} & \multicolumn{2}{|c|}{ Annotations } \\
\hline & & & & & & $3,4^{\prime}, 5$-Trihydroxystilbene; resveratrol & Seselin \\
\hline 13180 & 0.0006 & -3.138 & $\mathrm{C}_{28} \mathrm{H}_{22} \mathrm{O}_{6}$ & $\mathrm{Ph}$ & 453.134393 & Gnetin A & $\varepsilon$-Viniferin \\
\hline 8552 & 0.0029 & +2.979 & $\mathrm{C}_{10} \mathrm{H}_{18} \mathrm{~N}_{4} \mathrm{O}_{6}$ & $\mathrm{Pp}$ & 289.115364 & $\begin{array}{l}\text { N-(L-arginino)succinate; } \\
\text { 2-(nomega-L-arginino)succinate; } \\
\text { L-argininosuccinate; L-argininosuccinic } \\
\text { acid }\end{array}$ & $\begin{array}{l}\text { Argininosuccinic acid (see KEGG C03406); } \\
\text { 2-[amino-(4-amino-4-carboxy-butyl) } \\
\text { imino-methyl]aminobutanedioic acid }\end{array}$ \\
\hline 13140 & 0.0028 & +3.025 & $\mathrm{C}_{21} \mathrm{H}_{44} \mathrm{NO}_{7} \mathrm{P}$ & $\mathrm{Lp}$ & 452.278290 & $\begin{array}{l}\text { LysoPE(0:0/16:0); 1-hydroxy-2- } \\
\text { hexadecanoyl-sn-glycero-3- } \\
\text { phosphoethanolamine }\end{array}$ & $\begin{array}{l}\text { LysoPE(16:0/0:0); } \\
\text { 1-hexadecanoyl-2-hydroxy-sn- } \\
\text { glycero-3-phosphoethanolamine }\end{array}$ \\
\hline 6674 & 0.0008 & +3.040 & $\mathrm{C}_{11} \mathrm{H}_{14} \mathrm{O}_{4}$ & $\mathrm{Ph}$ & 209.081940 & Sinapyl alcohol; sinapoyl alcohol & $\begin{array}{l}\text { 3-Methyl-1-(2,4,6-trihydroxyphenyl) } \\
\text { butan-1-one }\end{array}$ \\
\hline 9257 & 0.0028 & +3.100 & $\mathrm{C}_{13} \mathrm{H}_{21} \mathrm{~N}_{3} \mathrm{O}_{6}$ & $\mathrm{Pp}$ & 314.135789 & Clavamycin D & \\
\hline 12659 & 0.0014 & +3.104 & $\mathrm{C}_{21} \mathrm{H}_{43} \mathrm{O}_{7} \mathrm{P}$ & $\mathrm{Lp}$ & 437.267420 & $\begin{array}{l}\text { LPA(0:0/18:0) (see KEGG C00416); } \\
\text { 2-octadecanoyl-glycero-3P }\end{array}$ & $\begin{array}{l}\text { LPA(18:0/0:0) (see KEGG C00416); } \\
\text { 1-octadecanoyl-glycero-3P }\end{array}$ \\
\hline 9037 & 0.0053 & +3.110 & $\mathrm{C}_{10} \mathrm{H}_{17} \mathrm{~N}_{3} \mathrm{O}_{6} \mathrm{~S}$ & $\mathrm{Pp}$ & 306.076520 & $\begin{array}{l}\text { Glutathione; 5-L-glutamyl-L-cysteinylglycine } \\
\gamma \text {-L-glutamyl-L-cysteinyl-glycine; GSH; rec }\end{array}$ & $\begin{array}{l}\text {; N-(N- } \gamma \text {-L-glutamyl-L-cysteinyl)glycine; } \\
\text { uced glutathione }\end{array}$ \\
\hline 12020 & 0.0048 & +3.184 & $\mathrm{C}_{25} \mathrm{H}_{48} \mathrm{O}_{4}$ & Lp & 411.348011 & $\begin{array}{l}\text { MG(0:0/22:1(13Z)/0:0); } \\
\text { 2-(13Z-docosenoyl)-glycerol }\end{array}$ & $\begin{array}{l}\text { MG(22:1(13Z)/0:0/0:0); } \\
\text { 1-(13Z-docosenoyl)-glycerol }\end{array}$ \\
\hline 10154 & 0.0019 & +3.184 & $\mathrm{C}_{20} \mathrm{H}_{38} \mathrm{O}_{4}$ & Lp & 341.269697 & Eicosanedioic acid & $\begin{array}{l}\text { PGF1a alcohol; } \\
\text { 1,9S,11R,15S-tetrahydroxy-13E-prostaene }\end{array}$ \\
\hline 7059 & 0.0004 & +3.244 & $\mathrm{C}_{9} \mathrm{H}_{14} \mathrm{~N}_{2} \mathrm{O}_{5}$ & $\mathrm{Pp}$ & 229.082987 & $\begin{array}{l}\text { Aspartyl-L-proline; }(2 \mathrm{~S})-1-[(2 \mathrm{~S})-2 \text {-amino-3-c } \\
\text { acid }\end{array}$ & arboxy-propanoyl]pyrrolidine-2-carboxylic \\
\hline 13611 & 0.0006 & +3.319 & $\mathrm{C}_{17} \mathrm{H}_{28} \mathrm{NO}_{14} \mathrm{R}$ & $\mathrm{Lp}$ & 470.151575 & $\mathrm{R}$ replaced by $\mathrm{H}$ in $\alpha-\mathrm{N}$-acetylneuraminyl-2, & 3- $\beta$-D-galactosyl-R \\
\hline 8394 & 0.0009 & +3.321 & $\mathrm{C}_{12} \mathrm{H}_{19} \mathrm{~N}_{3} \mathrm{O}_{5}$ & $\mathrm{Pp}$ & 284.125188 & Glycylprolylhydroxyproline & \\
\hline 10356 & 0.0022 & +3.337 & $\mathrm{C}_{18} \mathrm{H}_{21} \mathrm{ClN}_{2} \mathrm{O}_{3}$ & $\mathrm{Lp}$ & 347.116987 & $\begin{array}{l}\text { Compound VII; 5-(5-(6-chloro-4-(4,5-dihydr } \\
\text { pentyl)-3-methylisoxazole }\end{array}$ & -2-oxazolyl)phenoxy) \\
\hline 11306 & 0.0026 & +3.425 & $\mathrm{C}_{23} \mathrm{H}_{44} \mathrm{O}_{4}$ & Lp & 383.316655 & $\begin{array}{l}\mathrm{MG}(0: 0 / 20: 1(11 \mathrm{Z}) / 0: 0) \\
\text { 2-(11-eicosenoyl)-glycerol }\end{array}$ & $\begin{array}{l}\text { MG(20:1(11Z)/0:0/0:0); } \\
\text { 1-(11-eicosenoyl)-glycerol }\end{array}$ \\
\hline 6556 & 0.0025 & +3.439 & $\mathrm{C}_{10} \mathrm{H}_{18} \mathrm{O}_{4}$ & Lp & 201.113231 & Sebacic acid; decanedioic acid & Diethyl adipate; diethyl hexanedioate \\
\hline 8030 & 0.0002 & +3.468 & $\mathrm{C}_{17} \mathrm{H}_{34} \mathrm{O}_{2}$ & $\mathrm{Lp}$ & 269.248606 & Methyl palmitate & Heptadecanoic acid \\
\hline 9202 & 0.0024 & +3.715 & $\mathrm{C}_{14} \mathrm{H}_{19} \mathrm{NO}_{7}$ & Lp & 312.108877 & $\begin{array}{l}\text { Tyramine glucuronide (see KEGG C03033); } \\
\text { phenoxy]-3,4,5-trihydroxyoxane-2-carboxyl }\end{array}$ & $\begin{array}{l}\text { (2S,3S,4S,5R,6S)-6-[4-(2-aminoethyl) } \\
\text { c acid }\end{array}$ \\
\hline 7319 & 0.0037 & +3.735 & $\mathrm{C}_{13} \mathrm{H}_{25} \mathrm{NO}_{3}$ & $\mathrm{Lp}$ & 242.176162 & N-Undecanoylglycine & \\
\hline 8849 & 0.0011 & +3.789 & $\mathrm{C}_{17} \mathrm{H}_{32} \mathrm{O}_{4}$ & Lp & 299.222777 & $\begin{array}{l}\mathrm{MG}(0: 0 / 14: 1(9 \mathrm{Z}) / 0: 0) \\
\text { 2-(9Z-tetradecenoyl)-glycerol }\end{array}$ & $\begin{array}{l}\text { MG(14:1(9Z)/0:0/0:0); } \\
\text { 1-(9Z-tetradecenoyl)-glycerol }\end{array}$ \\
\hline 11529 & 0.0010 & +3.838 & $\mathrm{C}_{16} \mathrm{H}_{24} \mathrm{O}_{11}$ & NM & 391.124610 & Shanzhiside & \\
\hline 6527 & 0.0012 & +3.884 & $\mathrm{C}_{10} \mathrm{H}_{16} \mathrm{O}_{4}$ & Lp & 199.097596 & $\begin{array}{l}\text { cis-4-Decenedioic acid; (4Z)-dec-4-enedioic } \\
\text { acid }\end{array}$ & cis-4-Decenedioic acid \\
\hline 8390 & $<0.0001$ & +3.942 & $\mathrm{C}_{12} \mathrm{H}_{15} \mathrm{NO}_{7}$ & $\mathrm{Ph}$ & 284.077572 & Buchananine & \\
\hline 10808 & 0.0001 & +4.006 & $\mathrm{C}_{24} \mathrm{H}_{46} \mathrm{O}_{2}$ & Lp & 365.342494 & $\begin{array}{l}\text { (15Z)-Tetracosenoic acid; nervonic acid; } \\
\text { (Z)-15-tetracosenoic acid }\end{array}$ & 10,13-Dimethyl-11-docosyne-10,13-diol \\
\hline 10006 & $<0.0001$ & +4.148 & $\mathrm{C}_{20} \mathrm{H}_{36} \mathrm{O}_{4}$ & Lp & 339.254064 & $\begin{array}{l}\text { 15S-HpEDE; } \\
\text { 15S-hydroperoxy-11Z,13E-eicosadienoic } \\
\text { acid }\end{array}$ & $\begin{array}{l}\text { PGE1 alcohol; } \\
\text { 1,11R,15S-trihydroxy-13E-prosten-9-one }\end{array}$ \\
\hline 11754 & 0.0001 & +4.171 & $\mathrm{C}_{20} \mathrm{H}_{34} \mathrm{O}_{8}$ & Lp & 401.218068 & $\begin{array}{l}\text { 5S-HETE di-endoperoxide; } \\
\text { 5S,15S-dihydroxy-9S,11R,8S,12S-diperoxy }\end{array}$ & 6E,13E-eicosadienoic acid \\
\hline 6580 & $<0.0001$ & +4.522 & $\mathrm{C}_{11} \mathrm{H}_{12} \mathrm{~N}_{2} \mathrm{O}_{2}$ & $\mathrm{Pp}$ & 203,082615 & $\begin{array}{l}\text { L-Tryptophan; tryptophan; } \\
\text { (S)- } \alpha \text {-amino- } \beta \text {-(3-indolyl)-propionic acid }\end{array}$ & D-Tryptophan \\
\hline 10010 & 0.0015 & +4.592 & $\mathrm{C}_{17} \mathrm{H}_{11} \mathrm{NO}_{7}$ & $\mathrm{Ph}$ & 340,046275 & Aristolochic acid; aristolochic acid I & \\
\hline
\end{tabular}

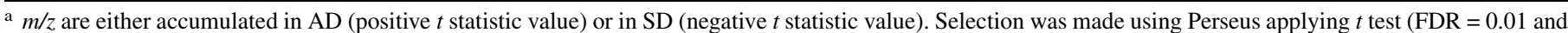
$\mathrm{S}_{0}=1$ ) and components are ranked according to their $t$ test statistic values. If multiple MassTrix annotations for the same formula were obtained, only the two first ones are listed. AD, white wood of diseased vines; SD, brown stripe of diseased vines; ID, component identity; $P$, $t$ test associated $P$ value; Cat., multidimensional stoichiometric constraints classification (MSCC) predicted structural category; amino sugars (As); phytochemicals (Ph); peptides (Pp); lipids (Lp); carbohydrates $(\mathrm{Cb})$; and $\mathrm{m} / z$, measured mass value. 
samples, whereas it was lipids (34\%) and peptides (21\%) in AD samples. Phytochemicals accumulated in SD included the phytoalexins resveratrol, $\varepsilon$-viniferin, piceatannol, and astringin. In AD samples, most accumulated lipids were phospholipids. As no toxin known to be produced by Botryosphaeriaceae was listed among discriminant compounds provided by statistical analysis, their presence was investigated in the global data file. Interestingly, putative terremutin hydrate $\left(\mathrm{m} / \mathrm{z}=173.0455679[\mathrm{M}-\mathrm{H}]^{-}\right)$, mellein $\left(\mathrm{m} / \mathrm{z}=177.0557238[\mathrm{M}-\mathrm{H}]^{-}\right)$, hydroxymellein $(\mathrm{m} / \mathrm{z}=193.0506336$ $\left.[\mathrm{M}-\mathrm{H}]^{-}\right)$, and succinate terremutin hydrate $\left(\mathrm{m} / \mathrm{z}=273.061045[\mathrm{M}-\mathrm{H}]^{-}\right)$ could be detected (Fig. 5). Their distribution was sample type- and cultivar-dependent. Chardonnay seemed to contain all these toxins in $\mathrm{AD}$ and, to a lesser extent, in SD area. Conversely, all toxins (except mellein) were more likely localized in SD for Gewurztraminer and Mourvèdre, while they were not detected (hydroxymellein for both cultivars; terremutin hydrate for Mourvèdre) or less accumulated in $\mathrm{AD}$ (succinate terremutin hydrate for both cultivars, terremutin hydrate for Gewurztraminer). Succinate terremutin hydrate was detected in all samples, even in AC of Mourvèdre. Similar investigation of resveratrol and $\varepsilon$-viniferin indicated that for the three cultivars, they were mostly accumulated in SD samples.

\section{DISCUSSION}

Spagnolo et al. (2014) used proteomic and targeted gene expression approaches to compare the impact of Botryosphaeria dieback infection on cultivars Chardonnay, Gewurztraminer (white cultivars), and Mourvèdre (red cultivar). They observed cultivardependent differences in the nature and abundance of proteins in the brown striped area (SD) and adjacent area (AD) of the trunk of diseased vines. In the present study, the same samples were analyzed by FT-ICR-MS to compare their metabolic fingerprints to deepen the characterization of this typical brown stripe associated to the Botryosphaeriaceae infection and adjacent asymptomatic area.

Samples were collected in July, i.e., during cambial functioning and before phellogen one. They exclusively consisted of wood tissue, i.e., mainly vessels surrounded by perivascular parenchyma and wood parenchyma (fibers). This tissue is characterized by a high degree of lignification of the cell walls. Vessels are dead cells but we cannot exclude that raw sap was partly collected during sampling. Biological isolation showed that cultivable Botryosphaeriaceae species were present in the wood area sampled,

A

Common CH GW [AC vs AD]
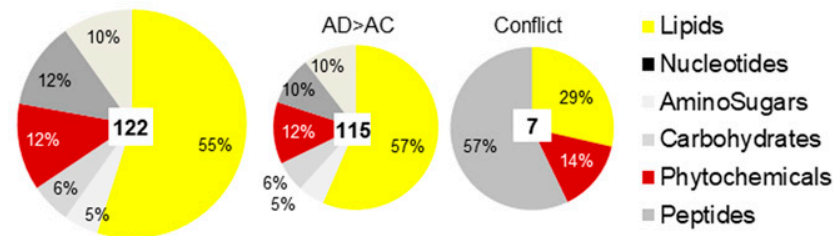

B

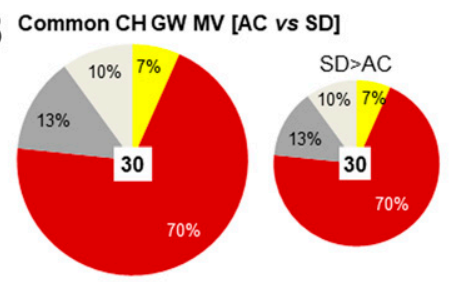

C

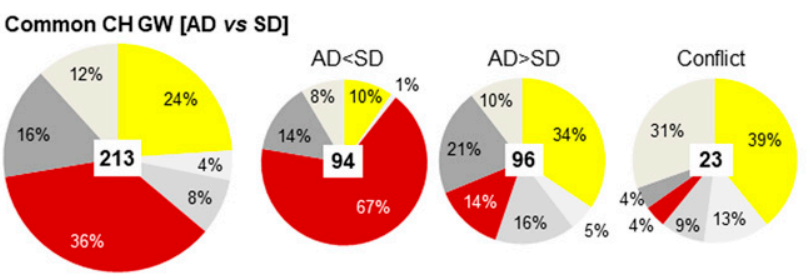

Fig. 4. Relative abundance $(\%)$ of the structural categories corresponding to formula obtained from the $\mathrm{m} / \mathrm{z}$ differently accumulated between sample types. The structural categories were predicted by multidimensional stoichiometric constraints classification on the filtered $\mathrm{m} / \mathrm{z}$ common to the three cultivars and resulting from $\mathbf{A}, \mathrm{AC}$ versus $\mathrm{AD}, \mathbf{B}, \mathrm{AC}$ versus $\mathrm{SD}$, and $\mathbf{C}, \mathrm{AD}$ versus $\mathrm{SD}$ comparisons. Conflict means reverse accumulation between sample types, depending on the cultivar. AC, white wood of asymptomatic vines; AD, white wood of diseased vines; SD, brown stripe of diseased vines; $\mathrm{CH}$, Chardonnay; GW, Gewurztraminer; MV, Mourvèdre; and NM, nonmatching structures.

TABLE 7. List of the 20 top $\mathrm{m} / z$ accumulated in Gewurztraminer SD samples compared with AD samples ${ }^{\mathrm{a}}$

\begin{tabular}{|c|c|c|c|c|c|c|c|}
\hline ID & $P$ & $t$ test & Formula & Cat. & $\mathrm{m} / \mathrm{z}$ & & otations \\
\hline 8690 & $<0.0001$ & -5.032 & $\mathrm{C}_{17} \mathrm{H}_{12} \mathrm{O}_{5}$ & $\mathrm{Ph}$ & 295.061199 & Aspulvinone E & $\begin{array}{l}\text { Pseudobaptigenin methyl ether; } \\
\text { 7-methoxy-3', } \\
\text { 4'-methylenedioxyisoflavone }\end{array}$ \\
\hline 8121 & $<0.0001$ & -4.186 & $\mathrm{C}_{15} \mathrm{H}_{14} \mathrm{O}_{5}$ & $\mathrm{Ph}$ & 273.076861 & Phloretin & $\alpha$-Pyrufuran \\
\hline 10782 & $<0.0001$ & -4.055 & $\mathrm{C}_{13} \mathrm{H}_{19} \mathrm{O}_{10} \mathrm{P}$ & $\mathrm{Ph}$ & 365.064466 & Salicin 6P & \\
\hline 9308 & $<0.0001$ & -4.044 & $\mathrm{C}_{17} \mathrm{H}_{16} \mathrm{O}_{6}$ & $\mathrm{Ph}$ & 315.087404 & Cajanol & Homoferreirin \\
\hline 6697 & $<0.0001$ & -4.040 & $\mathrm{C}_{14} \mathrm{H}_{12} \mathrm{O}_{2}$ & $\mathrm{Ph}$ & 211.076478 & $\begin{array}{l}\text { Benzoin; 2-hydroxy- } \\
\text { 1,2-diphenylethanone; } \\
\text { benzoin tincture }\end{array}$ & Pinosylvin \\
\hline 11533 & $<0.0001$ & -4.008 & $\mathrm{C}_{20} \mathrm{H}_{24} \mathrm{O}_{8}$ & $\mathrm{Ph}$ & 391.139864 & Eupatoroxin & 10-epi-Eupatoroxin \\
\hline 12369 & $<0.0001$ & -3.980 & $\mathrm{C}_{20} \mathrm{H}_{25} \mathrm{ClO}_{8}$ & $\mathrm{Ph}$ & 427.116559 & Eupachloroxin & \\
\hline 14026 & $<0.0001$ & -3.945 & $\mathrm{C}_{29} \mathrm{H}_{26} \mathrm{O}_{7}$ & $\mathrm{Ph}$ & 485.160643 & $\begin{array}{l}\text { Isogemichalcone B; } \\
\text { 3'-(4-coumaroyloxy- } \\
\text { 3-methylbutyl-2(E)- } \\
\text { enyl)- } 4,2^{\prime}, 4^{\prime} \text {-trihydroxychalcone }\end{array}$ & $\begin{array}{l}\text { Gemichalcone B; } \\
\text { 3'-(4-coumaroyloxy-3-methylbutyl-2(Z)- } \\
\text { enyl)-4,2',4'-trihydroxychalcone }\end{array}$ \\
\hline 9223 & $<0.0001$ & -3.898 & $\mathrm{C}_{17} \mathrm{H}_{14} \mathrm{O}_{6}$ & $\mathrm{Ph}$ & 313.071873 & Ventinone A & Irisolidone \\
\hline 13648 & 0.0005 & -3.830 & $\mathrm{C}_{30} \mathrm{H}_{48} \mathrm{O}_{4}$ & $\mathrm{Lp}$ & 471.348021 & Gratiogenin & Echinocystic acid \\
\hline 13274 & 0.0002 & -3.797 & $\mathrm{C}_{30} \mathrm{H}_{48} \mathrm{O}_{3}$ & $\mathrm{Lp}$ & 455.353111 & Betulinic acid & $\alpha$-Elemolic acid \\
\hline 15851 & 0.0001 & -3.692 & $\mathrm{C}_{27} \mathrm{H}_{32} \mathrm{O}_{15}$ & $\mathrm{Ph}$ & 595.167016 & Isobutrin & Butrin; butin 7,3'-di-O-glucoside \\
\hline 10621 & 0.0001 & -3.691 & $\mathrm{C}_{22} \mathrm{H}_{16} \mathrm{O}_{5}$ & $\mathrm{Ph}$ & 359.092546 & Pinocembrin 7-O-benzoate & \\
\hline 12549 & $<0.0001$ & -3.665 & $\mathrm{C}_{21} \mathrm{H}_{22} \mathrm{O}_{10}$ & $\mathrm{Ph}$ & 433.114055 & Naringenin $7-O-\beta$-D-glucoside; prunin & Phlorizin chalcone; chalcone 2'-O-glucoside \\
\hline 8424 & 0.0002 & -3.649 & $\mathrm{C}_{13} \mathrm{H}_{18} \mathrm{O}_{7}$ & $\mathrm{Ph}$ & 285.097974 & Salicin; salicoside & $\begin{array}{l}\text { Gastrodin; 4-(hydroxymethyl) } \\
\text { phenyl- } \beta \text {-D-glucopyranoside }\end{array}$ \\
\hline 8017 & 0.0001 & -3.625 & $\mathrm{C}_{16} \mathrm{H}_{14} \mathrm{O}_{4}$ & $\mathrm{Ph}$ & 269.081951 & $\begin{array}{l}\text { (+)-Medicarpin; 3-hydroxy- } \\
\text { 9-methoxypterocarpan }\end{array}$ & Vignafuran \\
\hline 11475 & 0.0003 & -3.605 & $\mathrm{C}_{20} \mathrm{H}_{22} \mathrm{O}_{8}$ & $\mathrm{Ph}$ & 389.124188 & $\begin{array}{l}\text { Piceid; polydatin; } 3,4,5 \text {-trihydroxystilbene- } \\
\text { 3- } \beta \text {-monoglucoside }\end{array}$ & Populin \\
\hline 12556 & 0.0001 & -3.594 & $\mathrm{C}_{22} \mathrm{H}_{26} \mathrm{O}_{9}$ & $\mathrm{Ph}$ & 433.150351 & Eleganin & Graminiliatrin \\
\hline 11862 & 0.0003 & -3.566 & $\mathrm{C}_{20} \mathrm{H}_{22} \mathrm{O}_{9}$ & $\mathrm{Ph}$ & 405.119108 & Astringin & Trichocarpin \\
\hline
\end{tabular}

a Selection was made using Perseus applying $t$ test (FDR $=0.005$ and $\left.\mathrm{S}_{0}=1\right)$. Components are ranked according to their $t$ test statistic values. If multiple MassTrix annotations for the same formula were obtained, only the two first ones are listed. AD, white wood of diseased vines; SD, brown stripe of diseased vines; ID, component identity; $P, t$ test associated $P$ value; Cat., multidimensional stoichiometric constraints classification (MSCC) predicted structural category; amino sugars (As); phytochemicals $(\mathrm{Ph})$; peptides $(\mathrm{Pp})$; lipids $(\mathrm{Lp})$; carbohydrates $(\mathrm{Cb})$; and $\mathrm{m} / \mathrm{z}$, measured mass value. 
TABLE 8. List of the common annotated $m / z$ differently accumulated in AC and AD, resulting from AC versus AD comparison $(t \text { test FDR }<0.05)^{\mathrm{a}}$

\begin{tabular}{|c|c|c|c|c|c|c|c|}
\hline ID & $\mathrm{CH} t$ test & GW $t$ test & Formula & Cat & $m / z$ & Annot & ations \\
\hline 11523 & -3.938 & -2.617 & $\mathrm{C}_{22} \mathrm{H}_{16} \mathrm{O}_{7}$ & $\mathrm{Ph}$ & 391.082331 & Hosloppin & 8-C-p-Hydroxybenzylkaempferol \\
\hline 7693 & -3.803 & -2.524 & $\mathrm{C}_{11} \mathrm{H}_{20} \mathrm{~N}_{2} \mathrm{O}_{5}$ & $\mathrm{Pp}$ & 259.129924 & L- $\gamma$-Glutamyl-L-isoleucine & L- $\gamma$-Glutamyl-L-leucine \\
\hline 9942 & -3.292 & -2.909 & $\mathrm{C}_{16} \mathrm{H}_{18} \mathrm{O}_{8}$ & $\mathrm{Ph}$ & 337.092840 & 1-Caffeoyl-4-deoxyquinic acid & 4-p-Coumaroylquinic acid \\
\hline 13981 & -3.121 & -2.855 & $\mathrm{C}_{21} \mathrm{H}_{24} \mathrm{O}_{13}$ & $\mathrm{Ph}$ & 483.114397 & Diospyrin & \\
\hline 8172 & -3.072 & -2.460 & $\mathrm{C}_{11} \mathrm{H}_{16} \mathrm{O}_{8}$ & $\mathrm{Ph}$ & 275.077231 & Ranunculin & \\
\hline 7433 & -3.072 & -2.500 & $\mathrm{C}_{10} \mathrm{H}_{17} \mathrm{NO}_{6}$ & $\mathrm{Lp}$ & 246.098303 & Linamarin & $\begin{array}{l}\text { Malonylcarnitine; (3S)-3-(3-hydroxy-3- } \\
\text { oxopropanoyl)oxy-4- } \\
\text { trimethylazaniumylbutanoate }\end{array}$ \\
\hline 17171 & -3.006 & -2.692 & $\mathrm{C}_{39} \mathrm{H}_{69} \mathrm{O}_{10} \mathrm{P}$ & $\mathrm{Lp}$ & 727.455621 & $\begin{array}{l}\text { PG(13:0/20:4(5Z,8Z,11Z,14Z)); 1- } \\
\text { tridecanoyl-2-(5Z,8Z,11Z,14Z- } \\
\text { eicosatetraenoyl)-glycero-3-phospho-(1'- } \\
\text { sn-glycerol) }\end{array}$ & $\begin{array}{l}\text { PG(15:0/18:4(6Z,9Z,12Z,15Z)); 1- } \\
\text { pentadecanoyl-2-(6Z,9Z,12Z,15Z- } \\
\text { octadecatetraenoyl)-glycero-3-phospho- } \\
\text { (1'-sn-glycerol) }\end{array}$ \\
\hline 11978 & -3.005 & -2.626 & $\mathrm{C}_{19} \mathrm{H}_{39} \mathrm{O}_{7} \mathrm{P}$ & $\mathrm{Lp}$ & 409.236062 & $\begin{array}{l}\text { 1-Palmitoylglycerol 3P; 1-hexadecanoyl-sn- } \\
\text { glycero-3P }\end{array}$ & $\begin{array}{l}\text { LPA(0:0/16:0) (see KEGG C00416); 2- } \\
\text { hexadecanoyl-glycero-3P }\end{array}$ \\
\hline 8145 & -2.982 & -2.499 & $\mathrm{C}_{10} \mathrm{H}_{13} \mathrm{NO}_{8}$ & $\mathrm{Ph}$ & 274.057019 & 3- $\beta$-D-Glucopyranuronosyloxy-5-methylisoxa & zole \\
\hline 12506 & -2.894 & -2.901 & $\mathrm{C}_{21} \mathrm{H}_{37} \mathrm{O}_{7} \mathrm{P}$ & $\mathrm{Lp}$ & 431.220429 & $\begin{array}{l}\text { PA(18:3(6Z,9Z,12Z)/0:0); 1-(6Z,9Z,12Z- } \\
\text { octadecatrienoyl)-glycero-3P }\end{array}$ & $\begin{array}{l}\mathrm{PA}(18: 3(9 \mathrm{Z}, 12 \mathrm{Z}, 15 \mathrm{Z}) / 0: 0) ; 1-(9 \mathrm{Z}, 12 \mathrm{Z}, 15 \mathrm{Z}- \\
\quad \text { octadecatrienoyl)-glycero-3P }\end{array}$ \\
\hline 12745 & -2.848 & -2.464 & $\mathrm{C}_{20} \mathrm{H}_{24} \mathrm{O}_{9} \mathrm{~S}$ & $\mathrm{Ph}$ & 439.106483 & Hallactone B & $\begin{array}{l}\mathrm{R} \text { replaced by } \mathrm{H} \text { in } 3,5,7,9,11,13,15,17- \\
\text { Octaoxo-eicosanoyl-[acp] }\end{array}$ \\
\hline 12566 & -2.838 & -2.683 & $\mathrm{C}_{21} \mathrm{H}_{39} \mathrm{O}_{7} \mathrm{P}$ & $\mathrm{Lp}$ & 433.236085 & Oleoylglycerone phosphate & $\begin{array}{l}\text { LPA(0:0/18:2(9Z,12Z)) (see KEGG } \\
\text { C00416); 2-(9Z,12Z-octadecadienoyl)- } \\
\text { glycero-3P }\end{array}$ \\
\hline 12192 & -2.783 & -2.910 & $\mathrm{C}_{17} \mathrm{H}_{26} \mathrm{O}_{12}$ & $\mathrm{Cb}$ & 421.135131 & Lamiide & 10-Hydroxymorroniside \\
\hline 6371 & -2.768 & -2.951 & $\mathrm{C}_{7} \mathrm{H}_{11} \mathrm{NO}_{5}$ & As & 188.056451 & $\begin{array}{l}\text { N-Acetyl-L-glutamate; N-acetyl-L-glutamic } \\
\text { acid }\end{array}$ & $\begin{array}{l}\text { L-2-Amino-6-oxoheptanedioate; L-2- } \\
\text { Amino-6-oxopimelate }\end{array}$ \\
\hline 17357 & -2.747 & -2.710 & $\mathrm{C}_{43} \mathrm{H}_{79} \mathrm{O}_{8} \mathrm{P}$ & $\mathrm{Lp}$ & 753.544006 & $\begin{array}{l}\text { PA(18:1(9Z)/22:2(13Z,16Z)); 1-(9Z- } \\
\text { octadecenoyl)-2-(13Z,16Z-docosadienoyl)- } \\
\text { glycero-3P }\end{array}$ & $\begin{array}{l}\text { PA(18:2(9Z,12Z)/22:1(11Z)); 1-(9Z,12Z- } \\
\text { octadecadienoyl)-2-(11Z-docosenoyl)- } \\
\text { glycero-3P }\end{array}$ \\
\hline 8347 & -2.696 & -2.698 & $\mathrm{C}_{12} \mathrm{H}_{17} \mathrm{NO}_{7}$ & $\mathrm{Lp}$ & 286.093239 & Volkenin & \\
\hline 11190 & -2.662 & -2.624 & $\mathrm{C}_{21} \mathrm{H}_{16} \mathrm{O}_{7}$ & $\mathrm{Ph}$ & 379.082660 & Diphyllin & Tetracenomycin F1 methylester \\
\hline 16981 & -2.624 & -2.625 & $\mathrm{C}_{37} \mathrm{H}_{71} \mathrm{O}_{10} \mathrm{P}$ & $\mathrm{Lp}$ & 705.471462 & $\begin{array}{l}\text { PG(12:0/19:1(9Z)); 1-dodecanoyl-2-(9Z- } \\
\text { nonadecenoyl)-glycero-3-phospho-( } 1^{\prime} \text {-sn- } \\
\text { glycerol) }\end{array}$ & $\begin{array}{l}\text { PG(13:0/18:1(9Z)); 1-tridecanoyl-2-(9Z- } \\
\text { octadecenoyl)-glycero-3-phospho-(1'-sn- } \\
\text { glycerol) }\end{array}$ \\
\hline 13994 & -2.570 & -2.744 & $\mathrm{C}_{22} \mathrm{H}_{45} \mathrm{O}_{9} \mathrm{P}$ & $\mathrm{Lp}$ & 483.272898 & PG(16:0/0:0); 1-hexadecanoyl-sn-glycero-3-p & hospho-(1'-sn-glycerol) \\
\hline 17882 & -2.535 & -2.520 & $\mathrm{C}_{43} \mathrm{H}_{79} \mathrm{O}_{13} \mathrm{P}$ & $\mathrm{Lp}$ & 833.519168 & $\begin{array}{l}\text { PI(16:0/18:2(9Z,12Z)) (see KEGG C00626); } \\
\text { 1-hexadecanoyl-2-(9Z,12Z- } \\
\text { octadecadienoyl)-sn-glycero-3- } \\
\text { phosphoinositol }\end{array}$ & $\begin{array}{l}\text { PI(16:1(9Z)/18:1(11Z)) (see KEGG } \\
\text { C00626); 1-(9Z-hexadecenoyl)-2-(11Z- } \\
\text { octadecenoyl)-sn-glycero-3- } \\
\text { phosphoinositol }\end{array}$ \\
\hline 17172 & -2.533 & -2.470 & $\mathrm{C}_{41} \mathrm{H}_{77} \mathrm{O}_{8} \mathrm{P}$ & $\mathrm{Lp}$ & 727.528590 & $\begin{array}{l}\text { PA(16:0/22:2(13Z,16Z)); 1-hexadecanoyl-2- } \\
\quad(13 Z, 16 Z \text {-docosadienoyl)-glycero-3P }\end{array}$ & $\begin{array}{l}\mathrm{PA}(16: 1(9 \mathrm{Z}) / 22: 1(11 \mathrm{Z})) ; 1-(9 \mathrm{Z}- \\
\text { hexadecenoyl)-2-(11Z-docosenoyl)- } \\
\text { glycero-3P }\end{array}$ \\
\hline 6804 & -2.513 & -2.503 & $\mathrm{C}_{8} \mathrm{H}_{13} \mathrm{NO}_{6}$ & As & 218.067023 & O-Succinyl-L-homoserine & N-Acetyl-D-mannosaminolactone \\
\hline 16418 & -2.479 & -2.714 & $\mathrm{C}_{36} \mathrm{H}_{65} \mathrm{O}_{8} \mathrm{P}$ & $\mathrm{Lp}$ & 655.434640 & $\begin{array}{l}\text { PA(13:0/20:3(8Z,11Z,14Z)); 1-tridecanoyl- } \\
\text { 2-(8Z,11Z,14Z-eicosatrienoyl)-glycero-3P }\end{array}$ & $\begin{array}{l}\text { PA(15:0/18:3(6Z,9Z,12Z)); 1- } \\
\text { pentadecanoyl-2-(6Z,9Z,12Z- } \\
\text { octadecatrienoyl)-glycero-3P }\end{array}$ \\
\hline 17860 & -2.477 & -2.668 & $\mathrm{C}_{43} \mathrm{H}_{77} \mathrm{O}_{13} \mathrm{P}$ & $\mathrm{Lp}$ & 831.503409 & $\begin{array}{l}\text { PI(14:0/20:3(8Z,11Z,14Z)); 1-tetradecanoyl- } \\
\text { 2-(8Z,11Z,14Z-eicosatrienoyl)-glycero-3- } \\
\text { phospho-(1'-myo-inositol) }\end{array}$ & $\begin{array}{l}\mathrm{PI}(14: 1(9 \mathrm{Z}) / 20: 2(11 \mathrm{Z}, 14 \mathrm{Z})) ; 1-(9 \mathrm{Z}- \\
\text { tetradecenoyl)-2-(11Z,14Z-eicosadienoyl)- } \\
\text { glycero-3-phospho-(1'-myo-inositol) }\end{array}$ \\
\hline 17364 & -2.476 & -2.681 & $\mathrm{C}_{43} \mathrm{H}_{81} \mathrm{O}_{8} \mathrm{P}$ & $\mathrm{Lp}$ & 755.560009 & $\begin{array}{l}\text { PA(18:0/22:2(13Z,16Z)); 1-octadecanoyl-2- } \\
\quad(13 Z, 16 Z \text {-docosadienoyl)-glycero-3P }\end{array}$ & $\begin{array}{l}\text { PA }(18: 1(9 Z) / 22: 1(11 Z)) ; 1-(9 Z- \\
\text { octadecenoyl)-2-(11Z-docosenoyl)- } \\
\text { glycero-3P }\end{array}$ \\
\hline 14298 & -2.469 & -2.650 & $\mathrm{C}_{21} \mathrm{H}_{20} \mathrm{O}_{14}$ & $\mathrm{Ph}$ & 495.078067 & Hibiscetin 3-glucoside & \\
\hline 16924 & -2.464 & -2.506 & $\mathrm{C}_{39} \mathrm{H}_{73} \mathrm{O}_{8} \mathrm{P}$ & $\mathrm{Lp}$ & 699.497295 & $\begin{array}{l}\text { PA(18:0/18:2(9Z,12Z)) (see KEGG } \\
\text { C00416); 1-octadecanoyl-2-(9Z,12Z- } \\
\text { octadecadienoyl)-sn-glycero-3P }\end{array}$ & $\begin{array}{l}\text { PA(18:1(11Z)/18:1(11Z)) (see KEGG } \\
\text { C00416); 1,2-di(11Z-octadecenoyl)-rac- } \\
\text { glycero-3P }\end{array}$ \\
\hline 16562 & -2.456 & -2.574 & $\mathrm{C}_{37} \mathrm{H}_{69} \mathrm{O}_{8} \mathrm{P}$ & $\mathrm{Lp}$ & 671.466004 & $\begin{array}{l}\text { PA(16:0/18:2(9Z,12Z)) (see KEGG } \\
\text { C00416); 1-hexadecanoyl-2-(9Z,12Z- } \\
\text { octadecadienoyl)-sn-glycero-3P }\end{array}$ & $\begin{array}{l}\text { PA(16:0/18:2(9Z,12Z)); 1-hexadecanoyl-2- } \\
\quad(9 Z, 12 Z \text {-octadecadienoyl)-sn-glycero-3P }\end{array}$ \\
\hline 16538 & -2.433 & -2.618 & $\mathrm{C}_{37} \mathrm{H}_{67} \mathrm{O}_{8} \mathrm{P}$ & $\mathrm{Lp}$ & 669.450309 & $\begin{array}{l}\text { PA(14:0/20:3(8Z,11Z,14Z)); 1- } \\
\text { tetradecanoyl-2-(8Z,11Z,14Z- } \\
\text { eicosatrienoyl)-glycero-3P }\end{array}$ & $\begin{array}{l}\mathrm{PA}(14: 1(9 \mathrm{Z}) / 20: 2(11 \mathrm{Z}, 14 \mathrm{Z})) ; 1-(9 \mathrm{Z}- \\
\text { tetradecenoyl)-2-(11Z,14Z-eicosadienoyl)- } \\
\text { glycero-3P }\end{array}$ \\
\hline 16184 & -2.406 & -2.481 & $\mathrm{C}_{34} \mathrm{H}_{65} \mathrm{O}_{8} \mathrm{P}$ & $\mathrm{Lp}$ & 631.434712 & $\begin{array}{l}\text { PA(12:0/19:1(9Z)); 1-dodecanoyl-2-(9Z- } \\
\text { nonadecenoyl)-glycero-3P }\end{array}$ & $\begin{array}{l}\text { PA(13:0/18:1(9Z)); 1-tridecanoyl-2-(9Z- } \\
\text { octadecenoyl)-glycero-3P }\end{array}$ \\
\hline 16871 & -2.389 & -2.523 & $\mathrm{C}_{39} \mathrm{H}_{69} \mathrm{O}_{8} \mathrm{P}$ & $\mathrm{Lp}$ & 695.466015 & $\begin{array}{l}\text { PA(16:0/20:4(5Z,8Z,11Z,14Z)); 1- } \\
\text { hexadecanoyl-2-(5Z,8Z,11Z,14Z- } \\
\text { eicosatetraenoyl)-sn-glycero-3P }\end{array}$ & $\begin{array}{l}\mathrm{PA}(14: 0 / 22: 4(7 \mathrm{Z}, 10 \mathrm{Z}, 13 \mathrm{Z}, 16 \mathrm{Z})) ; 1- \\
\text { tetradecanoyl-2-(7Z,10Z,13Z,16Z- } \\
\text { docosatetraenoyl)-glycero-3P }\end{array}$ \\
\hline 16853 & -2.370 & -2.536 & $\mathrm{C}_{39} \mathrm{H}_{67} \mathrm{O}_{8} \mathrm{P}$ & $\mathrm{Lp}$ & 693.450342 & $\begin{array}{l}\text { PA }(14: 1(9 Z) / 22: 4(7 Z, 10 Z, 13 Z, 16 Z)) ; 1-(9 Z- \\
\text { tetradecenoyl)-2-(7Z,10Z,13Z,16Z- } \\
\text { docosatetraenoyl)-glycero-3P }\end{array}$ & $\begin{array}{l}\text { PA(18:1(9Z)/18:4(6Z,9Z,12Z,15Z)); 1-(9Z- } \\
\text { octadecenoyl)-2-(6Z,9Z,12Z,15Z- } \\
\text { octadecatetraenoyl)-glycero-3P }\end{array}$ \\
\hline
\end{tabular}

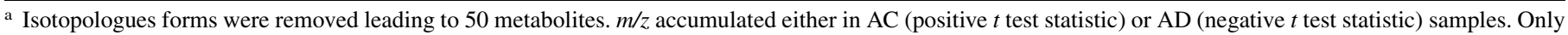
MassTrix two first annotations are mentioned. AC, white wood of asymptomatic vines; AD, white wood of diseased vines; ID, component identity; Chardonnay $(\mathrm{CH})$; Gewurztraminer (GW); Cat., multidimensional stoichiometric constraints classification (MSCC) predicted structural category; amino sugars (As); phytochemicals $(\mathrm{Ph})$; peptides $(\mathrm{Pp})$; lipids (Lp); carbohydrates $(\mathrm{Cb})$; and $\mathrm{m} / \mathrm{z}$, measured mass value.
} 
abundantly in SD samples and in lesser extent in AD samples (Spagnolo et al. 2014). AD areas were also colonized by other cultivable fungal species, whereas no-cultivable fungal species could be isolated from AC samples (Spagnolo et al. 2014). Overall, these results indicate that metabolites detected and identified in both SD and AD samples may be of plant or microbial origin.

FT-ICR-MS analysis allowed the detection of 6,499 raw mass values, which seems low compared with what could be detected in grapevine leaves of Chardonnay (Magnin-Robert et al. 2017). HCA of all samples (Supplementary Fig. S4) did not allow a distinction of cultivar or sample type (AC, $\mathrm{AD}$, and $\mathrm{SD}$ ) groups, making it necessary to dissociate sample sets for analysis.

The three cultivars were first considered separately for comparison of sample types. They were characterized by a similar number of $\mathrm{m} / \mathrm{z}$ (about 4,500) and structural category proportions (as defined by MSCC), with a majority of phytochemicals and lipids. Phytochemicals category, which corresponds to secondary metabolites, was expected since woody tissues are rich in lignin related metabolites (especially monolignols), tannins, and other organic components such as phenolic compounds and terpenes (Belgacem and Pizzi 2016). Although cellulose is an abundant wood component, the lipid category was more represented than the carbohydrate one. It can be explained by the fact that lipids ionize very well in MS analysis compared with carbohydrates (Boutegrabet et al. 2012).

Comparison of $\mathrm{AC}$ and $\mathrm{AD}$ samples highlighted important differences in the number of $\mathrm{m} / \mathrm{z}$ differently accumulated in the three cultivars studied: high for Chardonnay $(1,723)$, low for Gewurztraminer (229), and only one for Mourvèdre. These results suggest that the asymptomatic area close to the brown stripe is not impacted by infection in a similar manner for the three cultivars. The metabolome of $\mathrm{AD}$ area could be differently impacted for several reasons: either by different defense responses, or by an asymptomatic stage of fungal infection (hypothetical situation in Chardonnay and Gewurztraminer); or no plant response and no

TABLE 8. (Continued from previous page)

\begin{tabular}{|c|c|c|c|c|c|c|c|}
\hline ID & $\mathrm{CH} t$ test & GW $t$ test & Formula & Cat & $m / z$ & Anno & ations \\
\hline 16337 & -2.354 & -2.516 & $\mathrm{C}_{35} \mathrm{H}_{69} \mathrm{O}_{8} \mathrm{P}$ & Lp & 647.465942 & $\begin{array}{l}\text { PA(16:0/16:0) (see KEGG C00416); 1,2- } \\
\text { dihexadecanoyl-rac-glycero-3P }\end{array}$ & $\begin{array}{l}\mathrm{PA}(16: 0 / 16: 0)[\mathrm{rac}] ; 1,2 \text {-dihexadecanoyl-sn- } \\
\text { glycero-3P }\end{array}$ \\
\hline 18022 & -2.328 & -2.573 & $\mathrm{C}_{45} \mathrm{H}_{79} \mathrm{O}_{13} \mathrm{P}$ & $\mathrm{Lp}$ & 857.519267 & $\begin{array}{l}\text { PI(16:0/20:4(5Z,8Z,11Z,14Z)) (see KEGG } \\
\text { C00626); 1-hexadecanoyl-2- } \\
\text { (5Z,8Z,11Z,14Z-eicosatetraenoyl)-sn- } \\
\text { glycero-3-phosphoinositol }\end{array}$ & $\begin{array}{l}\text { PI(16:0/20:4(8Z,11Z,14Z,17Z)) (see KEGG } \\
\text { C00626); 1-hexadecanoyl-2- } \\
\text { (8Z,11Z,14Z,17Z-eicosapentaenoyl)-sn- } \\
\text { glycero-3-phosphoinositol }\end{array}$ \\
\hline 6859 & -2.305 & -2.709 & $\mathrm{C}_{13} \mathrm{H}_{18} \mathrm{O}_{3}$ & $\mathrm{Lp}$ & 221.118327 & $\begin{array}{l}\text { Dehydrovomifoliol; (6R)-6-hydroxy-3-oxo- } \\
\alpha \text {-ionone }\end{array}$ & $(+/-)-6$-Hydroxy-3-oxo- $\alpha$-ionone \\
\hline 16702 & -2.301 & -2.572 & $\mathrm{C}_{38} \mathrm{H}_{67} \mathrm{O}_{8} \mathrm{P}$ & $\mathrm{Lp}$ & 681.450280 & $\begin{array}{l}\mathrm{PA}(13: 0 / 22: 4(7 \mathrm{Z}, 10 \mathrm{Z}, 13 \mathrm{Z}, 16 \mathrm{Z})) ; 1- \\
\text { tridecanoyl-2-(7Z,10Z,13Z,16Z- } \\
\text { docosatetraenoyl)-glycero-3P }\end{array}$ & $\begin{array}{l}\text { PA }(15: 0 / 20: 4(5 Z, 8 Z, 11 Z, 14 Z)) ; 1- \\
\text { pentadecanoyl-2-(5Z,8Z,11Z,14Z- } \\
\text { eicosatetraenoyl)-glycero-3P }\end{array}$ \\
\hline 6414 & -2.300 & -2.768 & $\mathrm{C}_{7} \mathrm{H}_{12} \mathrm{O}_{6}$ & $\mathrm{Cb}$ & 191.056114 & $\begin{array}{l}\text { Quinate; quinic acid; kinic acid; chinic acid; } \\
\text { L-quinic acid; L-quinate; (-)-quinic acid }\end{array}$ & $\begin{array}{l}\text { 2D-5-O-Methyl-2,3,5/4,6- } \\
\text { pentahydroxycyclohexanone; 5D-5-O- } \\
\text { methyl-2,3,5/4,6- } \\
\text { pentahydroxycyclohexanone; D-5-O- } \\
\text { methyl-2,3,5/4,6- } \\
\text { pentahydroxycyclohexanone }\end{array}$ \\
\hline 16436 & -2.294 & -2.533 & $\mathrm{C}_{36} \mathrm{H}_{67} \mathrm{O}_{8} \mathrm{P}$ & $\mathrm{Lp}$ & 657.450319 & $\begin{array}{l}\text { PA(13:0/20:2(11Z,14Z)); 1-tridecanoyl-2- } \\
\quad(11 Z, 14 Z \text {-eicosadienoyl)-glycero-3P }\end{array}$ & $\begin{array}{l}\text { PA }(14: 1(9 Z) / 19: 1(9 Z)) ; 1-(9 Z- \\
\text { tetradecenoyl)-2-(9Z-nonadecenoyl)- } \\
\text { glycero-3P }\end{array}$ \\
\hline 17118 & -2.234 & -2.615 & $\mathrm{C}_{38} \mathrm{H}_{75} \mathrm{O}_{10} \mathrm{P}$ & $\mathrm{Lp}$ & 721.502885 & $\begin{array}{l}\text { PG(16:0/16:0); 1,2-dihexadecanoyl-sn- } \\
\text { glycero-3-phospho-(1'-sn-glycerol) }\end{array}$ & $\begin{array}{l}\text { PG(18:0/14:0); 1-octadecanoyl-2- } \\
\text { tetradecanoyl-sn-glycero-3-phospho-( } 1^{\prime} \text { - } \\
\text { sn-glycerol) }\end{array}$ \\
\hline 17801 & -2.188 & -2.733 & $\mathrm{C}_{42} \mathrm{H}_{79} \mathrm{O}_{13} \mathrm{P}$ & Lp & 821.518938 & $\begin{array}{l}\text { PI(13:0/20:1(11Z)); 1-tridecanoyl-2-(11Z- } \\
\text { eicosenoyl)-glycero-3-phospho-(1'-myo- } \\
\text { inositol) }\end{array}$ & $\begin{array}{l}\mathrm{PI}(14: 0 / 19: 1(9 \mathrm{Z})) ; 1 \text {-tetradecanoyl-2-(9Z- } \\
\text { nonadecenoyl)-glycero-3-phospho-(1'- } \\
\text { myo-inositol) }\end{array}$ \\
\hline 17340 & -2.160 & -2.618 & $\mathrm{C}_{40} \mathrm{H}_{79} \mathrm{O}_{10} \mathrm{P}$ & $\mathrm{Lp}$ & 749.534147 & $\begin{array}{l}\text { PG(16:0/18:0); 1-hexadecanoyl-2- } \\
\text { octadecanoyl-sn-glycero-3-phospho-(1'- } \\
\text { sn-glycerol) }\end{array}$ & $\begin{array}{l}\text { PG(18:0/16:0); 1-octadecanoyl-2- } \\
\text { hexadecanoyl-sn-glycero-3-phospho-( } 1^{\prime} \text { - } \\
\text { sn-glycerol) }\end{array}$ \\
\hline 17699 & -2.144 & -2.621 & $\mathrm{C}_{41} \mathrm{H}_{77} \mathrm{O}_{13} \mathrm{P}$ & $\mathrm{Lp}$ & 807.503428 & $\begin{array}{l}\text { PI(16:0/16:1(9Z)) (see KEGG C00626); } \\
\text { 1-hexadecanoyl-2-(9Z-hexadecenoyl)-sn- } \\
\text { glycero-3-phosphoinositol }\end{array}$ & $\begin{array}{l}\text { PI(16:1(9Z)/16:0) (see KEGG C00626); } \\
\text { 1-(9Z-hexadecenoyl)-2-hexadecanoyl-sn- } \\
\text { glycero-3-phosphoinositol }\end{array}$ \\
\hline 18042 & -2.139 & -2.815 & $\mathrm{C}_{45} \mathrm{H}_{83} \mathrm{O}_{13} \mathrm{P}$ & $\mathrm{Lp}$ & 861.550160 & $\begin{array}{l}\text { PI(16:0/20:2(11Z,14Z)) (see KEGG } \\
\text { C00626); 1-hexadecanoyl-2-(11Z,14Z- } \\
\text { eicosadienoyl)-sn-glycero-3- } \\
\text { phosphoinositol }\end{array}$ & $\begin{array}{l}\text { PI(18:0/18:2(9Z,12Z)) (see KEGG C00626); } \\
\text { 1-octadecanoyl-2-(9Z,12Z- } \\
\text { octadecadienoyl)-sn-glycero-3- } \\
\text { phosphoinositol }\end{array}$ \\
\hline 17301 & -2.100 & -2.652 & $\mathrm{C}_{40} \mathrm{H}_{75} \mathrm{O}_{10} \mathrm{P}$ & $\mathrm{Lp}$ & 745.502826 & $\begin{array}{l}\text { PG(16:0/18:2(9Z,12Z)); 1-hexadecanoyl-2- } \\
\text { (9Z,12Z-octadecadienoyl)-sn-glycero-3- } \\
\text { phospho-(1'-sn-glycerol) }\end{array}$ & $\begin{array}{l}\text { PG(16:1(9Z)/18:1(11Z)); 1-(9Z- } \\
\text { hexadecenoyl)-2-(11Z-octadecenoyl)-sn- } \\
\text { glycero-3-phospho-(1'-sn-glycerol) }\end{array}$ \\
\hline 17284 & -2.056 & -2.663 & $\mathrm{C}_{40} \mathrm{H}_{73} \mathrm{O}_{10} \mathrm{P}$ & $\mathrm{Lp}$ & 743.487156 & $\begin{array}{l}\text { PG(16:0/18:3(6Z,9Z,12Z)); 1-hexadecanoyl- } \\
\text { 2-(6Z,9Z,12Z-octadecatrienoyl)-sn- } \\
\text { glycero-3-phospho-(1'-sn-glycerol) }\end{array}$ & $\begin{array}{l}\text { PG(16:0/18:3(9Z,12Z,15Z)); 1- } \\
\text { hexadecanoyl-2-(9Z,12Z,15Z- } \\
\text { octadeatrienoyl)-sn-glycero-3-phospho- } \\
\text { (1'-sn-glycerol) }\end{array}$ \\
\hline 15855 & -2.013 & -2.459 & $\mathrm{C}_{27} \mathrm{H}_{49} \mathrm{O}_{12} \mathrm{P}$ & $\mathrm{Lp}$ & 595.289019 & PI(18:2(9Z,12Z)/0:0); 1-(9Z,12Z-octadecadie & noyl)-glycero-3-phospho-(1'-myo-inositol) \\
\hline 16999 & -2.012 & -2.680 & $\mathrm{C}_{37} \mathrm{H}_{73} \mathrm{O}_{10} \mathrm{P}$ & $\mathrm{Lp}$ & 707.487030 & $\begin{array}{l}\text { PG(12:0/19:0); 1-dodecanoyl-2- } \\
\text { nonadecanoyl-glycero-3-phospho-(1'-sn- } \\
\text { glycerol) }\end{array}$ & $\begin{array}{l}\text { PG(13:0/18:0); 1-tridecanoyl-2- } \\
\text { octadecanoyl-glycero-3-phospho-(1'-sn- } \\
\text { glycerol) }\end{array}$ \\
\hline 6580 & -4.254 & +2.506 & $\mathrm{C}_{11} \mathrm{H}_{12} \mathrm{~N}_{2} \mathrm{O}_{2}$ & $\mathrm{Pp}$ & 203.082615 & $\begin{array}{l}\text { L-Tryptophan; tryptophan; (S)- } \alpha \text {-amino- } \\
\beta \text {-(3-indolyl)-propionic acid }\end{array}$ & D-Tryptophan \\
\hline 6773 & -2.991 & +3.849 & $\mathrm{C}_{10} \mathrm{H}_{19} \mathrm{NO}_{4}$ & Lp & 216.124136 & O-Propanoylcarnitine; O-propionylcarnitine & $\begin{array}{l}\text { Propionylcarnitine (see KEGG C03017); } \\
\text { 3-propanoyloxy-4-trimethylammonio- } \\
\text { butanoate }\end{array}$ \\
\hline 13648 & -1.984 & +2.730 & $\mathrm{C}_{30} \mathrm{H}_{48} \mathrm{O}_{4}$ & $\mathrm{Lp}$ & 471.348021 & Gratiogenin & Echinocystic acid \\
\hline
\end{tabular}


asymptomatic stage of the fungal infection (hypothetical case in Mourvèdre). For Chardonnay and Gewurztraminer, the metabolites more accumulated in $\mathrm{AD}$ than in $\mathrm{AC}$ samples were mainly lipids, especially fatty acids for Chardonnay and the phospholipids phosphatidic acids (PA), phosphatidyl glycerol (PG), and phosphatidyl inositol (PI) for Gewurztraminer. Fatty acids are constituents of glycerolipids. They are now considered as signal molecules, especially jasmonic acid and traumatic acid (Farmer 1994), and play a key role in plant-pathogen interactions (Siebers et al. 2016; Walley et al. 2013). Jasmonic acid and traumatin were not listed among top $\mathrm{m} / \mathrm{z}$ but in the common $2,612 \mathrm{~m} / \mathrm{z}$ shared by the three cultivars (Supplementary File S1). The other fatty acids identified could be released from glycerolipids by lipases (Lee and Park 2019), but we cannot conclude if they are just a result of degradation or if they play a role in defense signaling. Salvatore et al. (2018) also detected fatty acids in the culture medium of $N$. parvum and $N$. vitifusiforme, suggesting their production and secretion. They only found linoleic, elaidic, and stearic acids for both fungi, whereas palmitoleic and palmitic acids were specifically found for $N$. vitifusiforme. Among them, only palmitic acid was listed among significant compounds in the present study. Phospholipids are major constituents in the biological membrane of organisms among which plants and fungi. They can also be found in lesser extent in fungi cell walls. Their higher abundance in AD samples suggests a fungal colonization of the wood, despite the absence of visible symptoms, which is consistent with the presence of fungi detected in this area. They may result, at least partially, from hydrolysis of membrane phospholipids by phospholipase D (PLD). PLD and its hydrolysis product phosphatidic acid are known for their role in plant immunity (for review, see Li and Wang 2019; Siebers et al. 2016; Zhang and Xiao 2015; Zhao 2015). In plant-pathogen interactions, the plant PLD is indeed activated after MAMP (microbe associated molecular pattern) or effector recognition and the released phosphatidic acid contributes to signaling of defense response (Hong et al. 2016). We can therefore hypothesize that Botryosphaeriaceae are perceived by grapevine and that defense responses in xylem parenchyma cells are in progress in $\mathrm{AD}$ area. Spagnolo et al. (2014) did not report PLD as overexpressed protein; the peak of PLD activity might have occurred prior to sampling time, making it nondiscriminant in their statistical analysis. Another possibility is that the identified lipid compounds could be the results of degradation by fungal action on wood composition. As reported by Esteves et al. (2014), fungi involved in Botryosphaeria dieback produced extracellular enzymes with lipase activity. Phytochemicals were also accumulated to a lesser extent, with for instance sinapyl alcohol, buchananine, hosloppin, and diphyllin. Sinapyl alcohol is a monolignol that can be incorporated into plant cell wall lignin polymer after oxidation by peroxidase and laccases (Cesarino et al. 2012). Its presence might contribute to cell wall strengthening, a defense response that is likely to limit infection (Sattler and Funnell-Harris 2013). It could result from plant peroxidase activity or from fungal laccase activity as it was reported that Botryosphaeriales fungi produce such enzymes (Esteves et al. 2014; Stempien et al. 2017). Buchananine is a pyridine alkaloid first identified in the stems of the climbing tree Cryptolepis buchanani (Dutta et al. 1978). Hosloppin is a pyrone-substituted flavonoid first identified in Hoslundia opposita (Ngadjui et al. 1995). Diphyllin was extracted from leaves of Cleistanthus collinus and its antimicrobial activity was demonstrated against Candida species (Suman et al. 2018). Since the occurrence of these four compounds in grapevine has never been reported, further specific studies would merit to be performed to definitely confirm their presence and study their role. Among them, it would be interesting to evaluate the antimicrobial activity of diphyllin against Botryosphaeriaceae.

Comparison between $\mathrm{AC}$ and $\mathrm{SD}$ samples also highlighted differences in the number of $\mathrm{m} / \mathrm{z}$ differently accumulated in the three cultivars studied: higher for Gewurztraminer $(1,701)$, intermediate for Mourvèdre (540), and lower for Chardonnay (64). Botryosphaeria dieback thus clearly impacts the metabolome of symptomatic area, regardless of the cultivar, and even for Mourvèdre for which no obvious significant difference could be highlighted for AC versus AD comparison. Most of $\mathrm{m} / \mathrm{z}$ values, which were more accumulated in SD samples compared with AC samples were predicted to be phytochemicals. Among them were the stilbenes resveratrol and $\varepsilon$-viniferin for Chardonnay and Mourvèdre, and also piceatannol, astringin, and piceid for Chardonnay. These stilbenic derivatives are grapevine phytoalexins, among which $\varepsilon$-viniferin, which has the highest antimicrobial activity (Adrian and Jeandet 2012; Adrian et al. 2012; Chong et al. 2009; Jeandet et al. 2002; Lambert et al. 2012). It was previously shown that metabolites secreted by $N$. parvum and D. seriata induce phytoalexin production and other defense responses in grapevine cell suspensions (Abou-Mansour et al. 2015; Stempien et al. 2018) and in plantlets (Trotel-Aziz et al.

TABLE 9. List of the common annotated $\mathrm{m} / \mathrm{z}$ differently accumulated in SD samples and resulting from AC versus SD comparison $(t \text { test FDR }<0.05)^{\mathrm{a}}$

\begin{tabular}{|c|c|c|c|c|c|c|c|c|}
\hline ID & $\begin{array}{c}\mathrm{CH} t \\
\text { test }\end{array}$ & $\begin{array}{c}\mathrm{GW} t \\
\text { test }\end{array}$ & $\begin{array}{c}\text { MV } t \\
\text { test }\end{array}$ & Formula & Cat. & $m / z$ & \multicolumn{2}{|c|}{ Annotations } \\
\hline 6977 & -4.709 & -2.679 & -1.932 & $\mathrm{C}_{14} \mathrm{H}_{12} \mathrm{O}_{3}$ & $\mathrm{Ph}$ & 227.071363 & $3,4^{\prime}, 5$-Trihydroxystilbene; resveratrol & Seselin \\
\hline 7347 & -3.432 & -2.390 & -1.949 & $\mathrm{C}_{14} \mathrm{H}_{12} \mathrm{O}_{4}$ & $\mathrm{Ph}$ & 243.066281 & $\begin{array}{l}3,3^{\prime}, 4^{\prime} 5 \text {-Tetrahydroxystilbene; } \\
\text { piceatannol }\end{array}$ & Wyerone acid \\
\hline 7635 & -7.962 & -1.961 & -2.365 & $\mathrm{C}_{14} \mathrm{H}_{10} \mathrm{O}_{5}$ & $\mathrm{Ph}$ & 257.045540 & $\begin{array}{l}\text { Gentisin; } \\
\text { 1,7-dihydroxy-3-methoxyxanthone }\end{array}$ & $\begin{array}{l}\text { Isogentisin; } \\
\text { 1,3-dihydroxy-7-methoxyxanthone }\end{array}$ \\
\hline 10738 & -3.366 & -3.108 & -2.285 & $\mathrm{C}_{21} \mathrm{H}_{16} \mathrm{O}_{6}$ & $\mathrm{Ph}$ & 363.087543 & Justicidin B & Robustone \\
\hline 13180 & -3.216 & -3.184 & -2.717 & $\mathrm{C}_{28} \mathrm{H}_{22} \mathrm{O}_{6}$ & $\mathrm{Ph}$ & 453.134393 & Gnetin A & $\varepsilon$-Viniferin \\
\hline 13584 & -3.074 & -3.290 & -2.470 & $\mathrm{C}_{28} \mathrm{H}_{22} \mathrm{O}_{7}$ & $\mathrm{Ph}$ & 469.129181 & Didymocalyxin B & \\
\hline 13995 & -3.012 & -2.920 & -2.444 & $\mathrm{C}_{30} \mathrm{H}_{44} \mathrm{O}_{5}$ & Lp & 483.311656 & Propapyriogenin A2 & $\begin{array}{l}\text { (25S)-3-oxo-12 } \beta \text { - } \\
\text { acetoxy-cholest-1,4-dien-26-oic acid }\end{array}$ \\
\hline 14026 & -3.159 & -3.934 & -2.728 & $\mathrm{C}_{29} \mathrm{H}_{26} \mathrm{O}_{7}$ & $\mathrm{Ph}$ & 485.160643 & $\begin{array}{l}\text { Isogemichalcone B; } \\
\text { 3'-(4-coumaroyloxy- } \\
\text { 3-methylbutyl- } \\
\text { 2(E)-enyl)- } 4,2^{\prime}, 4^{\prime} \text {-trihydroxychalcone }\end{array}$ & $\begin{array}{l}\text { Gemichalcone B; 3'-(4-coumaroyloxy- } \\
\text { 3-methylbutyl-2(Z)- } \\
\text { enyl)-4,2', } 4^{\prime} \text {-trihydroxychalcone }\end{array}$ \\
\hline 14261 & -3.114 & -2.935 & -2.351 & $\mathrm{C}_{22} \mathrm{H}_{22} \mathrm{O}_{13}$ & $\mathrm{Ph}$ & 493.098885 & $\begin{array}{l}\text { Pleurostimin 7-glucoside; } 5,6,7,3^{\prime}, 4^{\prime} \text { - } \\
\text { pentahydroxy-8-methoxyflavone } \\
\text { 7-glucoside }\end{array}$ & Laricitrin 3-galactoside \\
\hline 15952 & -3.116 & -2.233 & -2.417 & $\mathrm{C}_{32} \mathrm{H}_{30} \mathrm{O}_{12}$ & $\mathrm{Ph}$ & 605.166662 & $\begin{array}{l}\text { Skullcapflavone I } \\
2^{\prime}-\left(2^{\prime \prime}-\text { E-cinnamoylglucoside }\right)\end{array}$ & $\begin{array}{l}\text { Skullcapflavone I } \\
2^{\prime}-\left(4^{\prime \prime}-\text { E-cinnamoylglucoside }\right)\end{array}$ \\
\hline
\end{tabular}

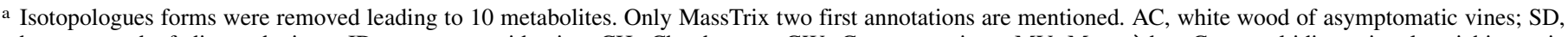
brown wood of diseased vines; ID, component identity; $\mathrm{CH}$, Chardonnay; GW, Gewurztraminer; MV, Mourvèdre; Cat., multidimensional stoichiometric constraints classification (MSCC) predicted structural category; amino sugars (As); phytochemicals (Ph); peptides (Pp); lipids (Lp); carbohydrates (Cb); and $m / z$, measured mass value. 
2019). Stilbene accumulation was also reported in the woody tissues of GTD-affected vines (Amalfitano et al. 2011; Amalfitano et al. 2000; Fontaine et al. 2016; Magnin-Robert et al. 2016). As far as Botryosphaeriaceae are concerned, Massonnet et al. (2018) detected stilbenes in the wood of Cabernet Sauvignon cuttings infected by N. parvum and Reis et al. (2016) in the green stems of Tempranillo cuttings infected by $N$. parvum or D. seriata. Spagnolo et al. (2014) also reported the presence of stilbene phytoalexins, together with overexpression of defense proteins, mainly PRproteins, in the brown striped area. Altogether, these results suggest

TABLE 10 . List of the common annotated $m / z$ differently accumulated in AD and SD, resulting from AD versus SD comparison $(t \text { test FDR }<0.05)^{\mathrm{a}}$

\begin{tabular}{|c|c|c|c|c|c|c|c|}
\hline ID & $\mathrm{CH} t$ test & $\mathrm{GW} t$ test & Formula & Cat. & $m / z$ & Anno & ations \\
\hline 6977 & -4.120 & -2.709 & $\mathrm{C}_{14} \mathrm{H}_{12} \mathrm{O}_{3}$ & $\mathrm{Ph}$ & 227.071363 & $3,4^{\prime}, 5$-Trihydroxystilbene; resveratrol & Seselin \\
\hline 13180 & -3.139 & -3.190 & $\mathrm{C}_{28} \mathrm{H}_{22} \mathrm{O}_{6}$ & $\mathrm{Ph}$ & 453.134393 & Gnetin A & $\varepsilon$-Viniferin \\
\hline 7347 & -3.070 & -2.429 & $\mathrm{C}_{14} \mathrm{H}_{12} \mathrm{O}_{4}$ & $\mathrm{Ph}$ & 243.066281 & $3,3^{\prime}, 4^{\prime} 5$-Tetrahydroxystilbene; piceatannol & Wyerone acid \\
\hline 14026 & -3.064 & -3.945 & $\mathrm{C}_{29} \mathrm{H}_{26} \mathrm{O}_{7}$ & $\mathrm{Ph}$ & 485.160643 & $\begin{array}{l}\text { Isogemichalcone } \mathrm{B} ; 3^{\prime}-(4-\text { coumaroyloxy-3- } \\
\text { methylbutyl-2(E)-enyl)-4,2', } 4^{\prime}- \\
\text { trihydroxychalcone }\end{array}$ & $\begin{array}{l}\text { Gemichalcone } \mathrm{B} ; 3^{\prime} \text {-(4-coumaroyloxy-3- } \\
\text { methylbutyl-2(Z)-enyl)- } 4,2^{\prime}, 4^{\prime}- \\
\text { trihydroxychalcone }\end{array}$ \\
\hline 13995 & -3.012 & -2.920 & $\mathrm{C}_{30} \mathrm{H}_{44} \mathrm{O}_{5}$ & $\mathrm{Lp}$ & 483.311656 & Propapyriogenin A2 & $\begin{array}{l}(25 \mathrm{~S})-3 \text {-Oxo-12 } \beta \text {-acetoxy-cholest-1,4-dien- } \\
26 \text {-oic acid }\end{array}$ \\
\hline 10738 & -2.890 & -3.089 & $\mathrm{C}_{21} \mathrm{H}_{16} \mathrm{O}_{6}$ & $\mathrm{Ph}$ & 363.087543 & Justicidin B & Robustone \\
\hline 16669 & -2.804 & -3.244 & $\mathrm{C}_{42} \mathrm{H}_{32} \mathrm{O}_{9}$ & $\mathrm{Lp}$ & 679.197769 & Canaliculatol & Copalliferol B \\
\hline 13274 & -2.784 & -3.797 & $\mathrm{C}_{30} \mathrm{H}_{48} \mathrm{O}_{3}$ & $\mathrm{Lp}$ & 455.353111 & Betulinic acid & $\alpha$-Elemolic acid \\
\hline 15952 & -2.640 & -2.234 & $\mathrm{C}_{32} \mathrm{H}_{30} \mathrm{O}_{12}$ & $\mathrm{Ph}$ & 605.166662 & $\begin{array}{l}\text { Skullcapflavone I 2'-(2"-E- } \\
\text { cinnamoylglucoside })\end{array}$ & $\begin{array}{l}\text { Skullcapflavone I 2'-(4"-E- } \\
\text { cinnamoylglucoside) }\end{array}$ \\
\hline 13584 & -2.631 & -3.219 & $\mathrm{C}_{28} \mathrm{H}_{22} \mathrm{O}_{7}$ & $\mathrm{Ph}$ & 469.129181 & Didymocalyxin B & \\
\hline 13905 & -2.560 & -2.001 & $\mathrm{C}_{23} \mathrm{H}_{28} \mathrm{O}_{11}$ & $\mathrm{Ph}$ & 479.156055 & Bruceine B & Isobrucein B \\
\hline 14261 & -2.365 & -2.779 & $\mathrm{C}_{22} \mathrm{H}_{22} \mathrm{O}_{13}$ & $\mathrm{Ph}$ & 493.098885 & $\begin{array}{l}\text { Pleurostimin } 7 \text {-glucoside; } 5,6,7,3^{\prime}, 4^{\prime} \text { - } \\
\text { pentahydroxy-8-methoxyflavone } 7 \text { - } \\
\text { glucoside }\end{array}$ & Laricitrin 3-galactoside \\
\hline 14603 & -2.345 & -2.888 & $\mathrm{C}_{32} \mathrm{H}_{48} \mathrm{O}_{5}$ & Lp & 511.342965 & Acetoxolone; acetylglycyrrhetinic acid & $\begin{array}{l}\text { ZK 159222; (5Z,7E,22E)-(1S,3R,24R)-25- } \\
\text { carbobutoxy-26,26-cyclo-9,10-seco- } \\
\text { 5,7,10(19),22-cholestatetraene-1,3,24-triol }\end{array}$ \\
\hline 13971 & -2.298 & -2.477 & $\mathrm{C}_{20} \mathrm{H}_{20} \mathrm{O}_{14}$ & $\mathrm{Ph}$ & 483.078086 & 1-O,6-O-Digalloyl- $\beta$-D-glucose & \\
\hline 13805 & -2.178 & -2.842 & $\mathrm{C}_{22} \mathrm{H}_{22} \mathrm{O}_{12}$ & $\mathrm{Ph}$ & 477.104045 & $\begin{array}{l}5,2^{\prime}, 4^{\prime}, 5^{\prime} \text {-Tetrahydroxy-7-methoxy-4- } \\
\text { phenylcoumarin 5-O-glucoside }\end{array}$ & Isorhamnetin 3-glucoside \\
\hline 9308 & -2.168 & -4.044 & $\mathrm{C}_{17} \mathrm{H}_{16} \mathrm{O}_{6}$ & $\mathrm{Ph}$ & 315.087404 & Cajanol & Homoferreirin \\
\hline 16464 & -2.159 & -2.544 & $\mathrm{C}_{37} \mathrm{H}_{58} \mathrm{O}_{10}$ & Lp & 661.396011 & \multicolumn{2}{|c|}{ 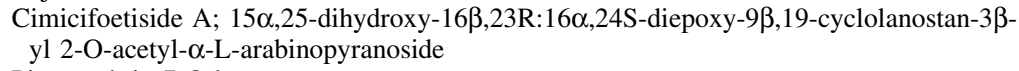 } \\
\hline 10621 & -2.120 & -3.691 & $\mathrm{C}_{22} \mathrm{H}_{16} \mathrm{O}_{5}$ & $\mathrm{Ph}$ & 359.092546 & Pinocembrin 7-O-benzoate & \\
\hline 12602 & -2.063 & -3.065 & $\mathrm{C}_{21} \mathrm{H}_{24} \mathrm{O}_{10}$ & $\mathrm{Ph}$ & 435.129714 & Phlorizin; phlorhizin; phloridzin & Epiafzelechin 3-O- $\beta$-D-allopyranoside \\
\hline 11862 & -2.056 & -3.566 & $\mathrm{C}_{20} \mathrm{H}_{22} \mathrm{O}_{9}$ & $\mathrm{Ph}$ & 405.119108 & Astringin & Trichocarpin \\
\hline 7635 & -2.015 & -2.756 & $\mathrm{C}_{14} \mathrm{H}_{10} \mathrm{O}_{5}$ & $\mathrm{Ph}$ & 257.045540 & Gentisin; 1,7-dihydroxy-3-methoxyxanthone & $\begin{array}{l}\text { Isogentisin; 1,3-dihydroxy-7- } \\
\text { methoxyxanthone }\end{array}$ \\
\hline 15851 & -2.002 & -3.692 & $\mathrm{C}_{27} \mathrm{H}_{32} \mathrm{O}_{15}$ & $\mathrm{Ph}$ & 595.167016 & Isobutrin & Butrin; butin 7,3'-di-O-glucoside \\
\hline 15976 & -1.992 & -2.471 & $\mathrm{C}_{27} \mathrm{H}_{28} \mathrm{O}_{16}$ & $\mathrm{Ph}$ & 607.130772 & Luteolin 7-glucuronide-4'-rhamnoside & $\begin{array}{l}\text { 6-Hydroxyluteolin 7-[3"-(3-hydroxy-3- } \\
\text { methylglutaryl)glucoside] }\end{array}$ \\
\hline 12259 & +2.053 & +2.298 & $\mathrm{C}_{20} \mathrm{H}_{41} \mathrm{O}_{7} \mathrm{P}$ & $\mathrm{Lp}$ & 423.251744 & \multirow{3}{*}{\multicolumn{2}{|c|}{$\begin{array}{l}\text { PA(17:0/0:0); 1-heptadecanoyl-glycero-3P } \\
\text { PG(22:2(13Z,16Z)/0:0); 1-(13Z,16Z-docosadienoyl)-glycero-3-phospho-(1'-sn-glycerol) } \\
\text { Blestriarene B }\end{array}$}} \\
\hline 15463 & +2.258 & +2.015 & $\mathrm{C}_{28} \mathrm{H}_{53} \mathrm{O}_{9} \mathrm{P}$ & $\mathrm{Lp}$ & 563.335503 & & \\
\hline 13904 & +2.321 & +2.381 & $\mathrm{C}_{30} \mathrm{H}_{24} \mathrm{O}_{6}$ & $\mathrm{Lp}$ & 479.150345 & & \\
\hline 13072 & +2.399 & +2.293 & $\mathrm{C}_{22} \mathrm{H}_{43} \mathrm{O}_{7} \mathrm{P}$ & $\mathrm{Lp}$ & 449.267381 & \multicolumn{2}{|c|}{ PA(19:1(9Z)/0:0); 1-(9Z-nonadecenoyl)-glycero-3P } \\
\hline 6414 & +2.412 & +2.094 & $\mathrm{C}_{7} \mathrm{H}_{12} \mathrm{O}_{6}$ & $\mathrm{Cb}$ & 191.056114 & $\begin{array}{l}\text { Quinate; quinic acid; kinic acid; chinic acid; } \\
\text { L-quinic acid; L-quinate; (-)-quinic acid }\end{array}$ & $\begin{array}{l}\text { 2D-5-O-methyl-2,3,5/4,6- } \\
\text { pentahydroxycyclohexanone; 5D-5-O- } \\
\text { methyl-2,3,5/4,6- } \\
\text { pentahydroxycyclohexanone; D-5-O- } \\
\text { methyl-2,3,5/4,6- } \\
\text { pentahydroxycyclohexanone }\end{array}$ \\
\hline 9942 & +2.702 & +1.938 & $\mathrm{C}_{16} \mathrm{H}_{18} \mathrm{O}_{8}$ & $\mathrm{Ph}$ & 337.092840 & 1-Caffeoyl-4-deoxyquinic acid & 4-p-Coumaroylquinic acid \\
\hline 16184 & +2.704 & +2.331 & $\mathrm{C}_{34} \mathrm{H}_{65} \mathrm{O}_{8} \mathrm{P}$ & $\mathrm{Lp}$ & 631.434712 & $\begin{array}{l}\text { PA(12:0/19:1(9Z)); 1-dodecanoyl-2-(9Z- } \\
\text { nonadecenoyl)-glycero-3P }\end{array}$ & $\begin{array}{l}\text { PA }(13: 0 / 18: 1(9 Z)) ; 1 \text {-tridecanoyl-2-(9Z- } \\
\text { octadecenoyl)-glycero-3P }\end{array}$ \\
\hline 8447 & +2.809 & +1.989 & $\mathrm{C}_{12} \mathrm{H}_{17} \mathrm{NO}_{7}$ & $\mathrm{Lp}$ & 286.093239 & \multicolumn{2}{|l|}{ Volkenin } \\
\hline 15031 & +2.817 & +2.211 & $\mathrm{C}_{18} \mathrm{H}_{32} \mathrm{O}_{18}$ & $\mathrm{Cb}$ & 535.151681 & \multicolumn{2}{|l|}{ 1,4-Beta-D-glucan (see KEGG C00760) } \\
\hline 16612 & +2.863 & +2.242 & $\mathrm{C}_{36} \mathrm{H}_{70} \mathrm{NO}_{8} \mathrm{P}$ & $\mathrm{Lp}$ & 674.476862 & $\begin{array}{l}\text { PC(14:0/14:1(9Z)) (see KEGG C00157); 1- } \\
\text { tetradecanoyl-2-(9Z-tetradecenoyl)-sn- } \\
\text { glycero-3-phosphocholine }\end{array}$ & $\begin{array}{l}\text { PC(14:1(9Z)/14:0) (see KEGG C00157); } \\
\text { 1-(9Z-tetradecenoyl)-2-tetradecanoyl- } \\
\text { sn-glycero-3-phosphocholine }\end{array}$ \\
\hline 16702 & +2.881 & +2.276 & $\mathrm{C}_{38} \mathrm{H}_{67} \mathrm{O}_{8} \mathrm{P}$ & $\mathrm{Lp}$ & 681.450280 & $\begin{array}{l}\mathrm{PA}(13: 0 / 22: 4(7 \mathrm{Z}, 10 \mathrm{Z}, 13 \mathrm{Z}, 16 \mathrm{Z})) ; 1- \\
\text { tridecanoyl-2-(7Z,10Z,13Z,16Z- } \\
\text { docosatetraenoyl)-glycero-3P }\end{array}$ & $\begin{array}{l}\text { PA(15:0/20:4(5Z,8Z,11Z,14Z)); 1- } \\
\text { pentadecanoyl-2-(5Z,8Z,11Z,14Z- } \\
\text { eicosatetraenoyl)-glycero-3P }\end{array}$ \\
\hline 12094 & +2.991 & +1.967 & $\mathrm{C}_{21} \mathrm{H}_{37} \mathrm{O}_{6} \mathrm{P}$ & Lp & 415.225543 & \multicolumn{2}{|c|}{ CPA(18:2(9Z,12Z)/0:0); 1-(9Z,12Z-octadecadienoyl)-glycero-3-cyclophosphate } \\
\hline 13994 & +3.021 & +2.031 & $\mathrm{C}_{22} \mathrm{H}_{45} \mathrm{O}_{9} \mathrm{P}$ & Lp & 483.272898 & \multicolumn{2}{|c|}{ PG(16:0/0:0); 1-hexadecanoyl-sn-glycero-3-phospho-(1'-sn-glycerol) } \\
\hline 12659 & +3.105 & +1.990 & $\mathrm{C}_{21} \mathrm{H}_{43} \mathrm{O}_{7} \mathrm{P}$ & Lp & 437.267420 & $\begin{array}{l}\text { LPA(0:0/18:0) (see KEGG C00416); 2- } \\
\text { octadecanoyl-glycero-3P }\end{array}$ & $\begin{array}{l}\text { LPA(18:0/0:0) (see KEGG C00416); } \\
\text { 1-octadecanoyl-glycero-3P }\end{array}$ \\
\hline 9037 & +3.111 & +1.945 & $\mathrm{C}_{10} \mathrm{H}_{17} \mathrm{~N}_{3} \mathrm{O}_{6} \mathrm{~S}$ & $\mathrm{Pp}$ & 306.076520 & \multicolumn{2}{|c|}{$\begin{array}{l}\text { Glutathione; 5-L-glutamyl-L-cysteinylglycine; N-(N- } \gamma \text {-L-glutamyl-L-cysteinyl)glycine; } \\
\gamma \text {-L-glutamyl-L-cysteinyl-glycine; GSH; reduced glutathione }\end{array}$} \\
\hline 11190 & +3.271 & +2.652 & $\mathrm{C}_{21} \mathrm{H}_{16} \mathrm{O}_{7}$ & $\mathrm{Ph}$ & 379.082660 & Diphyllin & Tetracenomycin F1 methylester \\
\hline
\end{tabular}

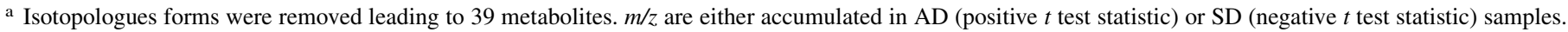
Only MassTrix two first annotations are mentioned. AD, white wood of diseased vines; SD, brown wood of diseased vines; ID, component identity; Chardonnay $(\mathrm{CH})$; Gewurztraminer (GW); Cat., multidimensional stoichiometric constraints classification (MSCC) predicted structural category; amino sugars (As); phytochemicals $(\mathrm{Ph})$; peptides $(\mathrm{Pp})$; lipids (Lp); carbohydrates $(\mathrm{Cb})$; and $\mathrm{m} / \mathrm{z}$, measured mass value. 
that cells of infected xylem parenchyma respond to the pathogens by immune responses.

Comparison between AD and SD also revealed specificity for Mourvèdre. For Chardonnay and Gewurztraminer, it confirmed a higher accumulation of lipids (mostly phospholipids) in AD samples and of phytochemicals (encompassing phytoalexins) in $\mathrm{SD}$ ones. In both $\mathrm{AC} / \mathrm{SD}$ and $\mathrm{AD} / \mathrm{SD}$ comparisons, resveratrol and its dimer $\varepsilon$-viniferin were listed as top $\mathrm{m} / \mathrm{z}$, for Chardonnay, but not for other cultivars. As Chardonnay is known to be less susceptible to GTDs than the two other cultivars analyzed (Bruez et al. 2013; Grosman and Doublet 2012; Murolo and Romanazzi 2014), this suggests that $\varepsilon$-viniferin contribute to this lower susceptibility. One can also not exclude the possible role of fatty acids that were found as Chardonnay significant top $\mathrm{m} / \mathrm{z}$ in diseased wood. Curiously, few phenolic compounds were listed among phytochemicals, although they have been reported to be accumulated in leaves of vines affected by GTDs (Lima et al. 2010, 2017; Moret et al. 2018), and in the wood of Botryosphaeria affected vines.
Some of them have antifungal activities and can cause alteration of hyphal morphology and inhibition of fungal ligninolytic activities (Gómez et al. 2016; Spagnolo et al. 2014). We can hypothesize that they are effectively present in samples, but not in high-enough concentrations for their selection as top $\mathrm{m} / \mathrm{z}$ by statistical analysis.

Amazingly, no difference was found between $\mathrm{AD}$ and SD samples for Mourvèdre, despite visually clear differences, at least in color. It is probably due to the huge sample heterogeneity between replicates of this cultivar, as revealed by HCA analysis (Supplementary Fig. S4). Hence, half (4/9) of the Mourvèdre SD samples demonstrated an over-accumulation of $\mathrm{m} / \mathrm{z}$ while the other half looked like AD ones. In the same manner, replicates of AC samples were for half of them (4/8) different in pattern. Altogether, only few compounds could be highlighted by statistical analysis on the Mourvèdre $m / z$ subset (Supplementary Fig. S2, step b). Further experimentation would merit to be conducted in order to check if this heterogeneity is cultivar dependent.
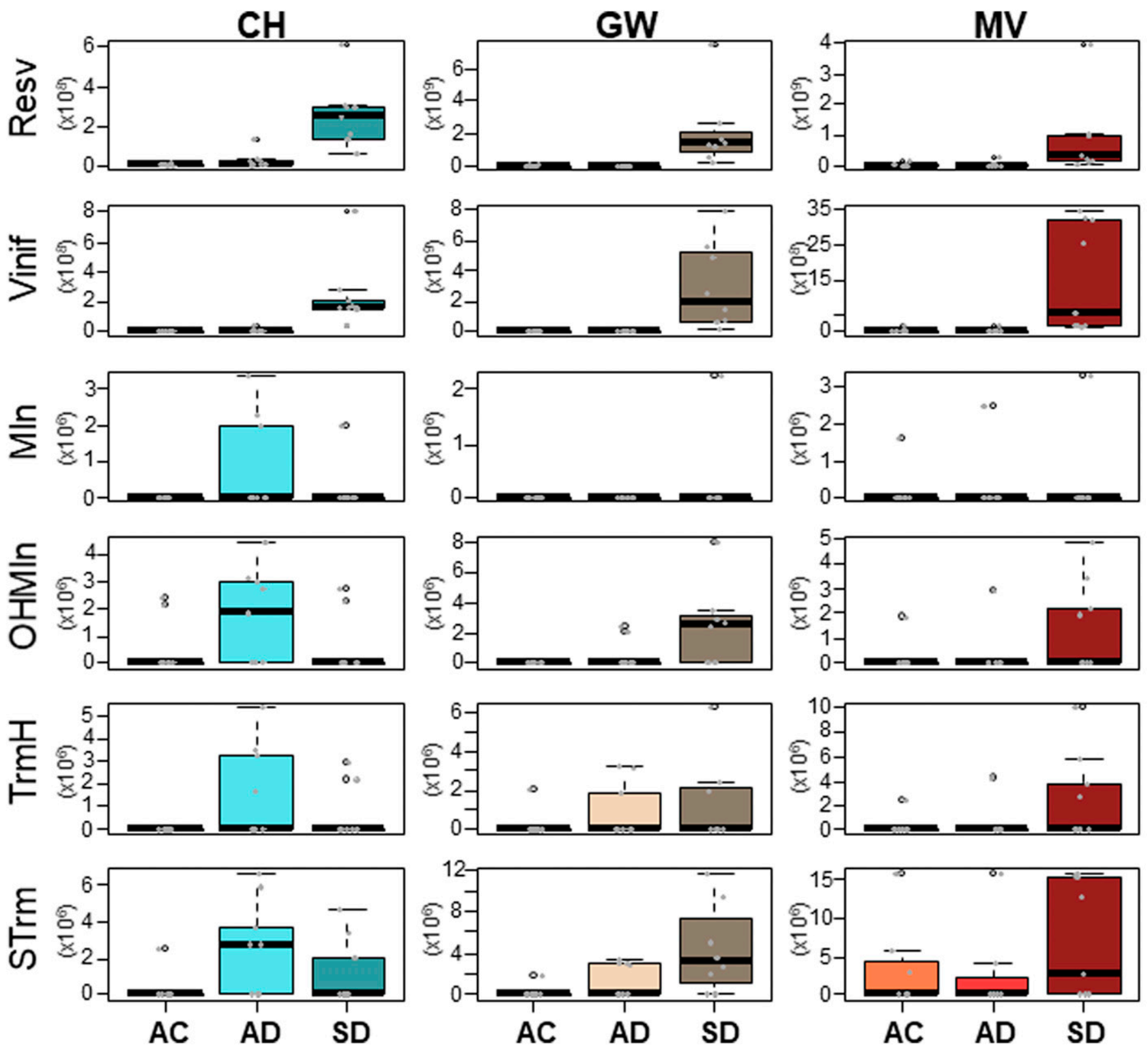

Fig. 5. Abundance of putative fungal toxins and vine phytoalexins resveratrol and $\varepsilon$-viniferin in the different sample types (AC, AD, and SD) for the three cultivars Chardonnay, Gewurztraminer, and Mourvèdre ( $\mathrm{CH}, \mathrm{GW}$, and MV, respectively). Values correspond to signal intensity. AC, white wood of asymptomatic vines; $\mathrm{AD}$, white wood of diseased vines; SD, brown stripe of diseased vines; resveratrol (Resv); viniferin (Vinif); mellein (Mln); hydroxy-mellein (OHMln); terremutin hydrate (TrmH); and succinate terremutin (STrm). 
It was demonstrated that the fungi associated to Botryosphaeria dieback secretes phytotoxins (Andolfi et al. 2011; Bellée et al. 2017; Benard-Gellon et al. 2015; Blanco-Ulate et al. 2013; Massonnet et al. 2017, 2018; Morales-Cruz et al. 2015). Investigation of the occurrence in the global data file edited in this study allowed us to find putative compounds of this family, including terremutin hydrate, mellein, hydroxymellein, and succinate terremutin hydrate. In the literature, attention is especially paid on these metabolites since their production could be associated to the fungal aggressiveness (Abou-Mansour et al. 2015; Benard-Gellon et al. 2015; Reveglia et al. 2019; Trotel-Aziz et al. 2019). Abou-Mansour et al. (2015) reported the occurrence of several toxins encompassing (-)-terremutin, (+)-terremutin hydrate, 4-hydroxysuccinateterremutin hydrate, $(R)-(-)$-mellein, and hydroxymellein. Curiously, we found putative terremutin and terremutin succinate that were previously reported as produced by $N$. parvum but not by D. seriata; despite only D. seriata was isolated from our samples (Spagnolo et al. 2014). As microbiological methods were used, the identification of $D$. seriata does not exclude the possibility that other Botryosphariaceae were present. Bruez et al. (2014) and Larignon et al. (2015) indeed showed that $N$. parvum and D. seriata, and in lesser extent $B$. dothidea, were frequently identified in French vineyards. In this study, the presence of the toxins was cultivar and sample type dependent. Succinate terremutin hydrate was detected in all samples, even in AC of Mourvèdre. This last observation would suggest that Mourvèdre control vines were probably also infected, despite the absence of visible symptoms. For succinate terremutin hydrate, it is the first report suggesting its presence in vine tissue since Abou-Mansour et al. (2015) only described this compound in in vitro cultures of N. parvum. However, these authors used 0.2 to $3 \mathrm{~g}$ of wood powder for analysis, and up to $0.5 \mathrm{~g}$ for Magnin-Robert et al. (2016), whereas only $0.15 \mathrm{~g}$ was used herein. Despite the high resolutive power of FT-ICR-MS, the amounts of the putative toxins might be under the level of detection in some samples, making it difficult to draw conclusions. It would be interesting to start a detailed study to specifically analyze Botryosphaeriaceae phytotoxins in AD and SD wood areas to have a better estimation of their abundance and spatial distribution. Interestingly, for Chardonnay, toxins were mostly accumulated in AD samples, whereas phytoalexins were mostly accumulated in SD samples, where cultivable Botryosphaeriaceae species were richly detected. This result suggests that the presence of phytoalexin is correlated with the presence of pathogens, and not with that of toxins. It also indicates that toxins are not accumulated in the brown stripe where there is maximal cultivable Botryosphaeriaceae species but in the adjacent asymptomatic area. Conversely, for Mourvèdre and Gewurztraminer, there is a colocalization of pathogens, toxins and stilbenes. This cultivar-dependent accumulation of toxins would merit deeper investigation. These results also indicate that phytoalexins are not sufficient to stop the biosynthesis of toxins by pathogens.

In a second time, the sample types $\mathrm{AC}, \mathrm{AD}$, and $\mathrm{SD}$ were compared and only the discriminant $\mathrm{m} / \mathrm{z}$, common to cultivars were selected for study. According to what was observed for the cultivardependent analysis, Mourvèdre was different, with neither $\mathrm{AC}$ versus $\mathrm{AD}$ nor $\mathrm{AD}$ versus $\mathrm{SD}$ discriminant $m / z$ common to Chardonnay and Gewurztraminer. As reported for the first comparison, lipids were more accumulated in AD samples whereas phytochemicals were more accumulated in SD samples, confirming that such evolution is not cultivar specific. Although specific markers of $\mathrm{AC}, \mathrm{AD}$, and $\mathrm{SD}$ areas could not be identified, our findings serve as a basis to draw attention to metabolic fingerprints of these areas.

Conclusion. Using a proteomic approach, Spagnolo et al. (2014) reported differential responses to Botryosphaeria dieback of Chardonnay, Gewurztraminer, and Mourvèdre. The present study shows that such differential responses are also expressed at the metabolome level. Phytochemicals and lipids were highlighted.
These results are original for lipids as this is the first time that a study reports the impact of Botryosphaeria dieback on wood phospholipid profiles.

\section{ACKNOWLEDGMENTS}

We thank the team of the Laboratory "Vigne, Biotechnologie et Environnement" from the University of Haute-Alsace and Philippe Larignon who helped to collect samples of Gewurztraminer and Mourvèdre, respectively; and Alessandro Spagnolo for his contribution to prepare samples.

\section{LITERATURE CITED}

Abou-Mansour, E., Debieux, J. L., Ramirez-Suero, M., Benard-Gellon, M., Magnin-Robert, M., Spagnolo, A., Chong, J., Farine, S., Bertsch, C., L'Haridon, F., Serrano, M., Fontaine, F., Rego, C., and Larignon, P. 2015. Phytotoxic metabolites from Neofusicoccum parvum, a pathogen of Botryosphaeria dieback of grapevine. Phytochemistry 115:207-215.

Adrian, M., and Jeandet, P. 2012. Effects of resveratrol on the ultrastructure of Botrytis cinerea conidia and biological significance in plant/pathogen interactions. Fitoterapia 83:1345-1350.

Adrian, M., Trouvelot, S., Gamm, M., Poinssot, B., Héloir, M., and Daire, X. 2012. Activation of grapevine defense mechanisms: Theoretical and applied approaches. Pages 313-331 in: Plant Defence: Biological Control. J. M. Mérillon and K. G. Ramawat, eds. Springer,Dordrecht, Netherlands.

Amalfitano, C., Agrelli, D., Arrigo, A., Mugnai, L., Surico, G., and Evidente, A. 2011. Stilbene polyphenols in the brown red wood of Vitis vinifera cv. Sangiovese affected by "Esca proper". Phytopathol. Mediterr. 50: S224-S235.

Amalfitano, C., Evidente, A., Surico, G., Tegli, S., Bertelli, E., and Mugnai, L. 2000. Phenols and stilbene polyphenols in the wood of Esca-diseased grapevines. Phytopathol. Mediterr. 39:178-183.

Andolfi, A., Mugnai, L., Luque, J., Surico, G., Cimmino, A., and Evidente, A. 2011. Phytotoxins produced by fungi associated with grapevine trunk diseases. Toxins (Basel) 3:1569-1605.

Belgacem, M., and Pizzi, A., eds. 2016. Lignocellulosic Fibers and Wood Handbook: Renewable Materials for Today's Environment. Wiley.

Bellée, A., Comont, G., Nivault, A., Abou-Mansour, E., Coppin, C., Dufour, M. C., and Corio-Costet, M. F. 2017. Life traits of four Botryosphaeriaceae species and molecular responses of different grapevine cultivars or hybrids. Plant Pathol. 66:763-776.

Benard-Gellon, M., Farine, S., Goddard, M. L., Schmitt, M., Stempien, E., Pensec, F., Laloue, H., Mazet-Kieffer, F., Fontaine, F., Larignon, P., Chong, J., Tarnus, C., and Bertsch, C. 2015. Toxicity of extracellular proteins from Diplodia seriata and Neofusicoccum parvum involved in grapevine Botryosphaeria dieback. Protoplasma 252:679-687.

Bertsch, C., Ramírez-Suero, M., Magnin-Robert, M., Larignon, P., Chong, J., Abou-Mansour, E., Spagnolo, A., Clément, C., and Fontaine, F. 2013. Grapevine trunk diseases: Complex and still poorly understood. Plant Pathol. 62:243-265.

Blanco-Ulate, B., Rolshausen, P., and Cantu, D. 2013. Draft genome sequence of Neofusicoccum parvum isolate UCR-NP2, a fungal vascular pathogen associated with grapevine cankers. Genome Announc. 1:e00339-13.

Boutegrabet, L., Kanawati, B., Gebefugi, I., Peyron, D., Cayot, P., Gougeon, R. D., and Schmitt-Kopplin, P. 2012. Attachment of chloride anion to sugars: Mechanistic investigation and discovery of a new dopant for efficient sugar ionization/detection in mass spectrometers. Chemistry 18: 13059-13067.

Bruez, E., Lecomte, P., Grosman, J., Doublet, B., Bertsch, C., Fontaine, F., Ugaglia, A., Teisedre, P. L., Da Costa, J. P., Guerin-Dubrana, L., and Rey, P. 2013. Overview of grapevine trunk diseases in France in the 2000s. Phytopathol. Mediterr. 52:262-275.

Bruez, E., Vallance, J., Gerbore, J., Lecomte, P., Da Costa, J.-P., Guerin-Dubrana, L., et al. 2014. Analyses of the temporal dynamics of fungal communities colonizing the healthy wood tissues of esca leafsymptomatic and asymptomatic vines. PLoS One 9:e95928.

Cesarino, I., Araújo, P., Domingues, A., Jr., and Mazzafera, P. 2012. An overview of lignin metabolism and its effect on biomass recalcitrance. Braz. J. Bot. 35:303-311.

Chong, J., Poutaraud, A., and Hugueney, P. 2009. Metabolism and roles of stilbenes in plants. Plant Sci. 177:143-155.

Dutta, S. K., Sharma, B. N., and Sharma, P. V. 1978. Buchananine, a novel pyridine alkaloid from Cryptolepis buchanani. Phytochemistry 17: 2047-2048.

Esteves, A. C., Saraiva, M., Correia, A., and Alves, A. 2014. Botryosphaeriales fungi produce extracellular enzymes with biotechnological potential. Can. J. Microbiol. 60:332-342. 
Farmer, E. E. 1994. Fatty acid signalling in plants and their associated microorganisms. Plant Mol. Biol. 26:1423-1437.

Fontaine, F., Pinto, C., Vallet, J., Clément, C., Gomes, A. C., and Spagnolo, A. 2016. The effects of grapevine trunk diseases (GTDs) on vine physiology. Eur. J. Plant Pathol. 144:707-721.

Gómez, P., Báidez, A. G., Ortuño, A., and Del Río, J. A. 2016. Grapevine xylem response to fungi involved in trunk diseases. Ann. Appl. Biol. 169: 116-124.

Gougeon, R. D., Lucio, M., De Boel, A., Frommberger, M., Hertkorn, N., Peyron, D., Chassagne, D., Feuillat, F., Cayot, P., Voilley, A., Gebefugi, I., and Schmitt-Kopplin, P. 2009. Expressing forest origins in the chemical composition of cooperage oak woods and corresponding wines by using FTICR-MS. Chemistry 15:600-611.

Grosman, J., and Doublet, B. 2012. Maladies du bois de la vigne: Synthèse des dispositifs d'observation au vignoble, de l'observatoire 2003-2008 au réseau d'épidémiosurveillance actuel. Phytoma-La Défense des végétaux 31-35.

Guerin-Dubrana, L., Fontaine, F., and Mugnai, L. 2019. Grapevine trunk disease in European and Mediterranean vineyards: occurrence, distribution and associated disease-affecting cultural factors. Phytopathol. Mediterr. 58:49-71.

Hofstetter, V., Buyck, B., Croll, D., Viret, O., Couloux, A., and Gindro, K. 2012. What if Esca disease of grapevine were not a fungal disease? Fungal Divers. 54:51-67.

Hong, Y., Zhao, J., Guo, L., Kim, S. C., Deng, X., Wang, G., Zhang, G., Li, M., and Wang, X. 2016. Plant phospholipases D and C and their diverse functions in stress responses. Prog. Lipid Res. 62:55-74.

Jeandet, P., Douillet-Breuil, A. C., Bessis, R., Debord, S., Sbaghi, M., and Adrian, M. 2002. Phytoalexins from the Vitaceae: Biosynthesis, phytoalexin gene expression in transgenic plants, antifungal activity, and metabolism. J. Agric. Food Chem. 50:2731-2741.

Lambert, C., Bisson, J., Waffo-Téguo, P., Papastamoulis, Y., Richard, T., Corio-Costet, M.-F., Mérillon, J.-M., and Cluzet, S. 2012. Phenolics and their antifungal role in grapevine wood decay: Focus on the Botryosphaeriaceae family. J. Agric. Food Chem. 60:11859-11868.

Larignon, P., Fulchic, R., Cere, L., and Dubos, B. 2001. Observation on black dead arm in French vineyards. Phytopathol. Mediterr. 40:336-342.

Larignon, P., Spagnolo, A., Bertsch, C., and Fontaine, F. 2015. First report of young grapevine decline caused by Neofusicoccum parvum in France. Plant Dis. 99:1859.

Lee, H.-J., and Park, O. K. 2019. Lipases associated with plant defense against pathogens. Plant Sci. 279:51-58.

Li, J., and Wang, X. 2019. Phospholipase D and phosphatidic acid in plant immunity. Plant Sci. 279:45-50.

Lima, M. R., Felgueiras, M. L., Graca, G., Rodrigues, J. E., Barros, A., Gil, A. M., and Dias, A. C. 2010. NMR metabolomics of Esca disease-affected Vitis vinifera cv. Alvarinho leaves. J. Exp. Bot. 61:4033-4042.

Lima, M. R. M., Felgueiras, M. L., Cunha, A., Chicau, G., Ferreres, F., and Dias, A. C. P. 2017. Differential phenolic production in leaves of Vitis vinifera cv. Alvarinho affected with Esca disease. Plant Physiol. Biochem. 112:45-52.

Magnin-Robert, M., Adrian, M., Trouvelot, S., Spagnolo, A., Jacquens, L., Letousey, P., Rabenoelina, F., Harir, M., Roullier-Gall, C., Clement, C., Schmitt-Kopplin, P., Vallat, A., Abou-Mansour, E., and Fontaine, F. 2017. Alterations in grapevine leaf metabolism occur prior to Esca apoplexy appearance. Mol. Plant-Microbe Interact. 30:946-959.

Magnin-Robert, M., Spagnolo, A., Boulanger, A., Joyeux, C., Clément, C., Abou-Mansour, E., and Fontaine, F. 2016. Changes in plant metabolism and accumulation of fungal metabolites in response to Esca proper and apoplexy expression in the whole grapevine. Phytopathology 106:541-553.

Massonnet, M., Figueroa-Balderas, R., Galarneau, E. R. A., Miki, S., Lawrence, D. P., Sun, Q., Wallis, C. M., Baumgartner, K., and Cantu, D. 2017. Neofusicoccum parvum colonization of the grapevine woody stem triggers asynchronous host responses at the site of infection and in the leaves. Front. Plant Sci. 8:1117.

Massonnet, M., Morales-Cruz, A., Figueroa-Balderas, R., Lawrence, D. P., Baumgartner, K., and Cantu, D. 2018. Condition-dependent co-regulation of genomic clusters of virulence factors in the grapevine trunk pathogen Neofusicoccum parvum. Mol. Plant Pathol. 19:21-34.

Mondello, V., Larignon, P., Armengol, J., Kortekamp, A., Vaczy, K., Prezman, F., Serrano, E., Rego, C., Mugnai, L., and Fontaine, F. 2018a. Management of grapevine trunk diseases: Knowledge transfer, current strategies and innovative strategies adopted in Europe. Phytopathol. Mediterr. 57:369-383.

Mondello, V., Songy, A., Battiston, E., Pinto, C., Coppin, C., Trotel-Aziz, P., Clement, C., Mugnai, L., and Fontaine, F. 2018b. Grapevine trunk diseases: A review of fifteen years of trials for their control with chemicals and biocontrol agents. Plant Dis. 102:1189-1217.

Morales-Cruz, A., Amrine, K. C., Blanco-Ulate, B., Lawrence, D. P., Travadon, R., Rolshausen, P. E., Baumgartner, K., and Cantu, D. 2015.
Distinctive expansion of gene families associated with plant cell wall degradation, secondary metabolism, and nutrient uptake in the genomes of grapevine trunk pathogens. BMC Genomics 16:469.

Moret, F., Lemaitre-Guillier, C., Grosjean, C., Clement, G., Coelho, C., Negrel, J., Jacquens, L., Morvan, G., Mouille, G., Trouvelot, S., Fontaine, F., and Adrian, M. 2018. Clone-dependent expression of esca disease revealed by leaf metabolite analysis. Front. Plant Sci. 9:1960.

Murolo, S., and Romanazzi, G. 2014. Effects of grapevine cultivar, rootstock and clone on Esca disease. Australas. Plant Pathol. 43:215-221.

Ngadjui, B. T., Tsopmo, A., Ayafor, J. F., Connolly, J. D., and Tamboue, H. 1995. Hosloppin, a new pyrone-substituted flavonoid from Hoslundia opposita. J. Nat. Prod. 58:109-111.

Reis, G. M., Faccin, H., Viana, C., Rosa, M. B., and de Carvalho, L. M. 2016. Vitis vinifera L. cv Pinot noir pomace and lees as potential sources of bioactive compounds. Int. J. Food Sci. Nutr. 67:789-796.

Reveglia, P., Masi, M., Cimmino, A., Michereff, S., Cinelli, T., Mugnai, L., and Evidente, A. 2019. Phytotoxins produced by Lasiodiplodia laeliocattleyae involved in Botryosphaeria dieback of grapevines in Brazil. Phytopathol. Mediterr. 58:207-211.

Rivas-Ubach, A., Liu, Y., Bianchi, T. S., Tolic, N., Jansson, C., and Pasa-Tolic, L. 2018. Moving beyond the van Krevelen diagram: A new stoichiometric approach for compound classification in organisms. Anal. Chem. 90: 6152-6160.

Romanazzi, G. 2009. Gray mold infection of Actinidia arguta in Italy. Plant Dis. 93:1221.

Rubio, J., and Garzón, E. 2011. Las enfermedades de madera de vid como amenaza del sector vitícola. Rev. Winetech 11:18-21.

Salvatore, M. M., Giambra, S., Naviglio, D., DellaGreca, M., Salvatore, F., Burruano, S., and Andolfi, A. 2018. Fatty acids produced by Neofusicoccum vitifusiforme and $N$. parvum, fungi associated with grapevine Botryosphaeria dieback. Agriculture 8:189.

Sattler, S. E., and Funnell-Harris, D. L. 2013. Modifying lignin to improve bioenergy feedstocks: Strengthening the barrier against pathogens? Front. Plant Sci. 4:70.

Siebers, M., Brands, M., Wewer, V., Duan, Y., Holzl, G., and Dormann, P. 2016. Lipids in plant-microbe interactions. Biochim. Biophys. Acta 9:27.

Spagnolo, A., Magnin-Robert, M., Alayi, T. D., Cilindre, C., Schaeffer-Reiss, C., Van Dorsselaer, A., Clement, C., Larignon, P., Ramirez-Suero, M., Chong, J., Bertsch, C., Abou-Mansour, E., and Fontaine, F. 2014. Differential responses of three grapevine cultivars to Botryosphaeria dieback. Phytopathology 104:1021-1035.

Stempien, E., Goddard, M. L., Leva, Y., Benard-Gellon, M., Laloue, H., Farine, S., Kieffer-Mazet, F., Tarnus, C., Bertsch, C., and Chong, J. 2018. Secreted proteins produced by fungi associated with Botryosphaeria dieback trigger distinct defense responses in Vitis vinifera and Vitis rupestris cells. Protoplasma 255:613-628.

Stempien, E., Goddard, M. L., Wilhelm, K., Tarnus, C., Bertsch, C., and Chong, J. 2017. Grapevine Botryosphaeria dieback fungi have specific aggressiveness factor repertory involved in wood decay and stilbene metabolization. PLoS One 12:e0188766.

Suman, T., Elangomathavan, R., Kasipandi, M., Chakkaravarthi, K., Tamilvendan, D., and Parimelazhagan, T. 2018. Diphyllin: An effective anticandidal agent isolated from Cleistanthus collinus leaf extract. Egypt. J. Basic Appl. Sci. 5:130-137.

Trotel-Aziz, P., Abou-Mansour, E., Courteaux, B., Rabenoelina, F., Clement, C., Fontaine, F., and Aziz, A. 2019. Bacillus subtilis PTA-271 counteracts Botryosphaeria dieback in grapevine, triggering immune responses and detoxification of fungal phytotoxins. Front. Plant Sci. 10:25.

Tziotis, D., Hertkorn, N., and Schmitt-Kopplin, P. 2011. Kendrick-analogous network visualisation of ion cyclotron resonance Fourier transform mass spectra: Improved options for the assignment of elemental compositions and the classification of organic molecular complexity. Eur. J. Mass Spectrom. (Chichester, Eng.) 17:415-421.

Urbez-Torres, J. 2011. The status of Botryosphaeriaceae species infecting grapevines. Phytopathol. Mediterr. 50:S5.

van Niekerk, J. M., Crous, P. W., Groenewald, J., Fourie, P. H., and Halleen, F. 2004. DNA phylogeny, morphology and pathogenicity of Botryosphaeria species on grapevines. Mycologia 96:781-798.

van Niekerk, J. M., Fourie, P. H., Halleen, F., and Crous, P. W. 2006. Botryosphaeria spp. as grapevine trunk disease pathogens. Phytopathol. Mediterr. 45:S43-S54.

Walley, J. W., Kliebenstein, D. J., Bostock, R. M., and Dehesh, K. 2013. Fatty acids and early detection of pathogens. Curr. Opin. Plant Biol. 16:520-526.

Zhang, Q., and Xiao, S. 2015. Lipids in salicylic acid-mediated defense in plants: Focusing on the roles of phosphatidic acid and phosphatidylinositol 4-phosphate. Front. Plant Sci. 6:387.

Zhao, J. 2015. Phospholipase D and phosphatidic acid in plant defence response: From protein-protein and lipid-protein interactions to hormone signalling. J. Exp. Bot. 66:1721-1736. 